DESY 07-061

\title{
Inflaton Decay in Supergravity
}

\author{
Motoi Endo ${ }^{1}$, Fuminobu Takahashi ${ }^{1}$, and T. T. Yanagida ${ }^{2,3}$ \\ 1 Deutsches Elektronen Synchrotron DESY, \\ Notkestrasse 85, 22607 Hamburg, Germany \\ ${ }^{2}$ Department of Physics, University of Tokyo, Tokyo 113-0033, Japan \\ ${ }^{3}$ Research Center for the Early Universe, \\ University of Tokyo, Tokyo 113-0033, Japan
}

\begin{abstract}
We discuss inflaton decay in supergravity, taking account of the gravitational effects. It is shown that, if the inflaton has a nonzero vacuum expectation value, it generically couples to any matter fields that appear in the superpotential at the tree level, and to any gauge sectors through anomalies in the supergravity. Through these processes, the inflaton generically decays into the supersymmetry breaking sector, producing many gravitinos. The inflaton also directly decays into a pair of the gravitinos. We derive constraints on both inflation models and supersymmetry breaking scenarios for avoiding overproduction of the gravitinos. Furthermore, the inflaton naturally decays into the visible sector via the top Yukawa coupling and $\mathrm{SU}(3)_{C}$ gauge interactions.
\end{abstract}




\section{INTRODUCTION}

Inflation [1] provides a simple solution to a number of serious shortcomings in the big bang cosmology such as the horizon and flatness problems. Above all, it can account for the origin of density fluctuations necessary to form the rich structure of our universe. In fact, the standard slow-roll inflation predicts almost scale-invariant power spectrum, which fits the recent cosmic microwave background (CMB) data [2] quite well.

It is now recognized that the universe underwent an inflationary epoch at an early stage. During the inflation, the universe is dominated by the potential energy of the inflaton, and experiences exponential expansion [1, 3]. After inflation ends, the inflaton field releases its energy into a thermal plasma by the decay, and the universe is reheated. Since all the particles including photons and baryons in the present universe are ultimately originated from the inflaton decay, it is of great importance to reveal how the reheating proceeds.

So far however, the reheating process has not been fully investigated. One often simplifies the whole reheating processes, and expresses them in terms of a single parameter, the reheating temperature. That is, the inflaton is assumed to have some ad hoc interactions with lighter degrees of freedom, i.e., the standard model (SM) particles in most cases, while possible productions of the hidden fields and/or gravitinos are neglected without definite grounds. However, many cosmological phenomena, e.g., baryogenesis, and production of dark matter and unwanted relics, crucially depend on the details of the reheating. Although the reheating temperature is certainly an important characteristic parameter, such simplification is too crude to truly describe cosmological scenarios.

Recently there has been much progress concerning the decays of scalar fields such as moduli [4, 5, 6] and inflaton [7, 8, 9, 10, 11] in a framework of the local supersymmetry (SUSY), i.e., the supergravity (SUGRA). The supersymmetric extension is one of the most promising candidates for the theory beyond SM. If SUSY exists at the TeV scale, the inflaton dynamics is quite likely described in SUGRA. In addition, since the existence of a flat direction is mediocre in SUSY models, one can find extremely flat potentials appropriate for the slow-roll inflation. Throughout this paper we consider inflation models in SUGRA. We have investigated the reheating of the universe in this framework, and found that the gravitinos are generically produced from the inflaton decay in most inflation models. In

particular, Ref. [7] has first pointed out that the inflaton can directly decay into a pair of 
the gravitinos. Moreover, incorporating the gravitational effects, Refs. [9, 11] have shown that the inflaton generically decays into the SUSY breaking sector, which produces the gravitinos (in)directly. The gravitino production rates due to these processes depend on the inflaton parameters as well as the detailed structure of the SUSY breaking sector. Such gravitino production clearly goes beyond the simplification of the reheating that has been adopted so far, and interestingly enough, it provides severe constraints on inflation models as well as the SUSY breaking scenarios. These constraints, together with the future collider experiments and observations on CMB, should become an important guide to understand the high energy physics and the early universe. The purpose of the present paper is to provide a global picture of the inflaton decay processes in SUGRA, paying special attention to the gravitino production. In particular, we explain which decay processes become most important under which circumstances. Not only do we summarize the decay processes found so far but we also give complete results on the spontaneous decay and the anomaly-induced decay processes, including the higher dimensional terms in the Kähler potential and the Kähler and sigma-model anomalies.

The organization of the paper is as follows. In Sec. II we review the gravitino pair production at the inflaton decay. Then we discuss the spontaneous decay at the tree level in Sec. III. In Sec. IV we consider the anomaly-induced decay of the inflaton, which proceeds via the anomalies in SUGRA. We provide some results on the decay rates, by way of illustration, for the minimal and sequestered Kähler potentials there. In Sec. V, we study cosmological implications of the decay processes explained in the preceding sections, particularly focusing on the constraints on the inflation models. The last section is devoted to conclusion.

\section{DECAY INTO A PAIR OF GRAVITINOS}

Once the inflaton field obtains a finite vacuum expectation value (VEV), it necessarily decays into the gravitinos. In this section, we briefly discuss the production of a pair of the gravitinos, and provide the partial decay rate. The process we consider is a perturbative decay, and the gravitinos are produced directly from the inflaton. The gravitino production 
is represented by the following interactions in the SUGRA Lagrangian [12] a;

$$
\begin{aligned}
e^{-1} \mathcal{L}= & \frac{1}{4} \epsilon^{k \ell m n}\left(G_{i} \partial_{k} \phi^{i}-G_{i^{*}} \partial_{k} \phi^{* i}\right) \bar{\psi}_{\ell} \bar{\sigma}_{m} \psi_{n} \\
& -\frac{1}{2} e^{G / 2}\left(G_{i} \phi^{i}+G_{i^{*}} \phi^{* i}\right)\left[\psi_{m} \sigma^{m n} \psi_{n}+\bar{\psi}_{m} \bar{\sigma}^{m n} \bar{\psi}_{n}\right],
\end{aligned}
$$

where $\sigma^{m n}=\frac{1}{4}\left(\sigma^{m} \bar{\sigma}^{n}-\sigma^{n} \bar{\sigma}^{m}\right)$, and we have chosen the unitary gauge in the Einstein frame. The sum over the indices is understood unless otherwise stated. We have also adopted the Planck unit $M_{P}=1\left(M_{P}=2.4 \times 10^{18} \mathrm{GeV}\right)$ here and in what follows unless it is written explicitly. The 2-spinor, $\psi_{m}$ ( or $\psi_{3 / 2}$ ), represents the gravitino, while $\phi^{i}$ collectively denotes an arbitrary scalar field including the inflaton $\phi$. Then the decay rate of the inflaton into a pair of the gravitinos, $\phi \rightarrow 2 \psi_{3 / 2}$, is evaluated as [4]

$$
\Gamma^{\text {(grav) }} \simeq \frac{\left|G_{\phi}\right|^{2}}{288 \pi} \frac{m_{\phi}^{5}}{m_{3 / 2}^{2} M_{P}^{2}},
$$

where $m_{3 / 2}=e^{G / 2}$ and $m_{\phi}$ are the masses of the gravitino and the inflaton, respectively. We readily find that the decay amplitude is inversely proportional to $m_{3 / 2}$. This is a result of an enhancement $\left(\propto m_{3 / 2}^{-2}\right)$ due to the longitudinal mode of the gravitino, $\psi_{m}(k) \propto k_{m} / m_{3 / 2} \sim$ $m_{\phi} / m_{3 / 2}$, which is partially compensated by the chirality suppression of the amplitude $\left(\propto m_{3 / 2}\right)$.

The decay amplitude crucially depends on $G_{\phi}$, which is a derivative of the generalized Kähler potential, $G=K+\ln |W|^{2}$, with respect to the inflaton field $\phi$. It is related to an $F$-term of the inflaton supermultiplet through the equation of motion, $F^{i}=-e^{G / 2} g^{i j^{*}} G_{j^{*}}$. In order to evaluate $G_{\phi}$, we need to incorporate the SUSY breaking field, $z$, into our analysis. This is because of the following reason. The decay is treated in the mass-eigenstate basis. In this basis, $\phi$ generally mixes with $z$ due to the SUGRA effects, unless the inflaton is protected by some symmetries which are preserved at the vacuum. We take $G_{z}=O(1)$ to have the vanishing cosmological constant. Then, $G_{z}$ can contribute to $G_{\phi}$ effectively via mixings between $\phi$ and $z$, which enhances gravitino production rate from inflaton decay. That is, the inflaton first oscillates into $z^{(*)}$, which then decays into a pair of the gravitinos: $\phi \rightleftharpoons z^{(*)} \rightarrow 2 \psi_{3 / 2}$.

a Due to the Kähler invariance, the generalized Kähler potential $G$ is more convenient and transparent than using the Kähler potential $K$ and the superpotential $W$. Since these two frames are related by the Weyl transformation, any physical amplitudes are equivalent at the tree level. 
The mixing angle depends on the mass spectrum of $\phi$ and $z$. The direct pair-gravitino production is effective especially for $m_{\phi} \ll m_{z}$. Such a large $m_{z}$ is often realized in the dynamical SUSY breaking (DSB) scenario [13]. In this case, there is a soft mass term, $K \sim|z|^{4} / \Lambda^{2}$ ( $\Lambda$ is the dynamical scale), and a scalar mass of $z$ can be larger than $m_{\phi}$, depending on inflation models. Then $G_{\phi}$ is given by [6]

$$
\left|G_{\phi}\right|^{2} \simeq\left|\sqrt{3} g_{\phi z^{*}}\right|^{2}+\left|\sqrt{3}\left(\nabla_{\phi} G_{z}\right) \frac{m_{3 / 2}}{m_{\phi}}\right|^{2}
$$

where we have neglected interference terms and higher dimensional operators in the Kähler potential. Here $g_{i j^{*}}=\frac{\partial^{2} K}{\partial \phi^{i} \partial \phi^{* j}}$ and $\nabla_{i} G_{j}=G_{i j}-\Gamma_{i j}^{k} G_{k}$ with $\Gamma_{j k}^{i}=g^{i \ell^{*}} g_{j \ell^{*} k}$. Note that the first term is from the mixing in the kinetic terms, while the SUGRA effects contribute to the second one. Thus even if there is no direct coupling between the inflaton and SUSYbreaking sectors in the global SUSY limit, the inflaton decays into a pair of the gravitinos for $m_{\phi} \ll m_{z}$.

Since each term of (3) is expected to depend on $\phi$ linearly, it is convenient to express the mixings as

$$
\begin{aligned}
\left|\nabla_{\phi} G_{z}\right| & \equiv c\langle\phi\rangle, \\
\left|g_{\phi z^{*}}\right| & \equiv \tilde{c}\langle\phi\rangle .
\end{aligned}
$$

In SUGRA, $c$ is estimated to be $O(1)$ for a generic Kähler potential by using $G_{z}=O(1)$, while $\tilde{c}$ depends on details of the SUSY breaking sector such as the VEV $\langle z\rangle$, e.g. $\tilde{c}=\langle z\rangle$ for $\delta K=|\phi|^{2}|z|^{2}$. Then, if $\tilde{c}$ is suppressed as in case of the minimal Kähler potential (i.e. $\left.g_{\phi z^{*}}=0\right)$, the gravitino pair production rate is

$$
\Gamma^{\text {(grav) }} \simeq \frac{c^{2}}{96 \pi}\left(\frac{\langle\phi\rangle}{M_{P}}\right)^{2} \frac{m_{\phi}^{3}}{M_{P}^{2}} .
$$

On the other hand, if the kinetic mixing is large, the rate is much enhanced as

$$
\Gamma^{\text {(grav) }} \simeq \frac{\tilde{c}^{2}}{96 \pi}\left(\frac{\langle\phi\rangle}{M_{P}}\right)^{2} \frac{m_{\phi}^{5}}{m_{3 / 2}^{2} M_{P}^{2}} .
$$

Such large gravitino production rates are cosmologically disastrous, which will be discussed in Sec. IV.

For high-scale inflation models with $m_{\phi} \gg m_{z}$, the pair-gravitino production rate depends on the detailed structure of the SUSY breaking models. If the SUSY breaking field is 
singlet and elementary above $\Lambda^{\mathrm{b}} \mathrm{c}$, the inflaton still directly decays into a pair of the gravitinos. In this case the relevant contribution to $G_{\phi}$ comes from higher dimensional

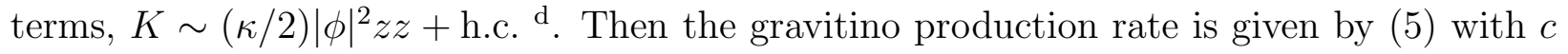
replaced with $\kappa$ (see Ref. [6, 7] for details). On the other hand, if the SUSY breaking field is composed of other fields and if the dynamical scale $\Lambda$ is below $m_{\phi}$, the direct production of the gravitinos becomes suppressed. Instead, as discussed in the following sections, gravitational effects force the inflaton to decay into the SUSY breaking sector.

Finally let us make a comment. In addition to the pair-gravitino production, the gravitino may be singly produced at the decay. This is the case when the inflaton mass comes from the soft SUSY breaking terms. Then the rate becomes as large as that given by (6) with $\tilde{c}=O(1)$. However, if the inflaton mass is provided by a SUSY-invariant mass term (as in most inflation models), such a single-gravitino production is negligible.

\section{SPONTANEOUS DECAY}

In this section we review the spontaneous decay of the inflaton, $\phi$, at the tree level. If the reheating is induced by the inflaton decay through non-renormalizable interactions, the reheating temperature can be low enough to satisfy the constraints from gravitinos produced by thermal scatterings [18, 19]. Since the interactions are then quite weak, the SUGRA effects may play an important role. Indeed, it has been recently pointed out that the SUGRA effects induce the inflaton decay [9]. The relevant channels of the inflaton decay contains the 2- and 3-body final states ${ }^{\mathrm{e}}$.

For the matter-fermion production, the relevant interactions are provided in the Einstein frame as [12]

$$
\begin{aligned}
e^{-1} \mathcal{L}= & -i g_{i j^{*}} \bar{\chi}^{j} \bar{\sigma}^{\mu} \partial_{\mu} \chi^{i} \\
& +\frac{1}{4} g_{i j^{*}} i\left(K_{k} \partial_{\mu} \phi^{k}-K_{k^{*}} \partial_{\mu} \phi^{* k}\right) \bar{\chi}^{j} \bar{\sigma}^{\mu} \chi^{i}-i g_{i j^{*}} \Gamma_{k \ell}^{i}\left(\partial_{\mu} \phi^{k}\right) \bar{\chi}^{j} \bar{\sigma}^{\mu} \chi^{\ell}
\end{aligned}
$$

b Such a singlet SUSY breaking field is necessary for the gauginos to have a sizable mass in the gravitymediated SUSY breaking scenario [14, 15]. See also Ref. 16] for a retrofitted gravity-mediation.

c In the DSB scenarios, the Polonyi problem was once solved since the $z$ field decays much before BBN due to a large soft scalar mass of $z[14,15]$. However, it has been recently found that the presence of such a field still puts a severe bound on the inflation scale [17].

d The contribution from this operator is suppressed when $m_{\phi}$ is smaller than $m_{z}[\underline{6}]$.

e Although $\phi$ may decay into 4-scalars, it is suppressed by the phase space and can be neglected. 


$$
-\frac{1}{2} e^{K / 2}\left(\mathcal{D}_{i} D_{j} W\right) \chi^{i} \chi^{j}+\text { h.c. }
$$

where $\mathcal{D}_{i} D_{j} W=W_{i j}+K_{i j} W+K_{i} D_{j} W+K_{j} D_{i} W-K_{i} K_{j} W-\Gamma_{i j}^{k} D_{k} W$ with $D_{i} W=$ $W_{i}+K_{i} W$. On the other hand, the matter-scalar production is represented by the kinetic term and the scalar potential;

$$
\begin{aligned}
e^{-1} \mathcal{L}= & -g_{i j^{*}} \partial_{\mu} \phi^{i} \partial^{\mu} \phi^{* j} \\
& -e^{K}\left[g^{i j^{*}}\left(D_{i} W\right)\left(D_{j} W\right)^{*}-3|W|^{2}\right] .
\end{aligned}
$$

In this paper, we adopt notation that, when the inflaton $\phi$ is explicitly shown in expressions, $\phi^{i}$ and $\chi^{i}$ represent only the matter fields. (We also use $Q$ to denote the matter fields.) Otherwise, as mentioned before, $\phi^{i}$ collectively denotes an arbitrary field including the inflaton $\phi$.

First let us consider decay processes induced by higher dimensional operators. The relevant one arises from such terms in the Kähler potential that holomorphically depend on the matter fields, $Q, Q^{\prime}$, i.e. $\delta K \sim|\phi|^{2} Q Q^{\prime}+$ h.c. ${ }^{\text {f }}$. The presence of such operators strongly depends on symmetries of the visible/hidden sectors. In Eq. (7) the term including $\Gamma_{i j}^{\phi}$ in the fermion mass is given by

$$
\mathcal{L}=\frac{1}{2} e^{K / 2} g_{\phi^{*} i j} g^{\phi \phi^{*}} W_{\phi \phi} \phi \chi^{i} \chi^{j}+\text { h.c. },
$$

which induces the inflaton decay into the two fermions $\left(\phi \rightarrow \bar{\chi}^{i} \bar{\chi}^{j}\right)$. Note here that $e^{K / 2} g^{\phi \phi^{*}} W_{\phi \phi} \equiv m_{\phi}$ is the inflaton mass.

On the other hand, the decay into the two scalars $\left(\phi \rightarrow \phi^{i} \phi^{j}\right)$ arises from the kinetic term of the matter scalars,

$$
\mathcal{L}=\frac{1}{2} g_{\phi i^{*} j^{*}}\left(\partial^{2} \phi\right) \phi^{* i} \phi^{* j}+\text { h.c.. }
$$

Using the equation of motion, $\partial^{2} \phi=m_{\phi}^{2} \phi$, one finds that the decay rates satisfy $\Gamma(\phi \rightarrow$ $\left.\phi^{i} \phi^{j}\right) \simeq \Gamma\left(\phi \rightarrow \bar{\chi}^{i} \bar{\chi}^{j}\right)$. The total rate then becomes

$$
\begin{aligned}
\Gamma^{(2-\text { body;hol })} & \equiv \Gamma\left(\phi \rightarrow \bar{\chi}^{i} \bar{\chi}^{j}\right)+\Gamma\left(\phi \rightarrow \phi^{i} \phi^{j}\right) \\
& \simeq \frac{\left|g_{\phi^{*} i j}\right|^{2}}{8 \pi} m_{\phi}\left(1-\frac{4 M_{Q}^{2}}{m_{\phi}^{2}}\right)^{\frac{1}{2}}
\end{aligned}
$$

\footnotetext{
f The decay process from this operator is obtained also in the global SUSY models.
} 
where $M_{Q}$ is a mass of the final state particles. Here note that $i$ and $j$ are fixed and the sum is not taken over these variables in the last expression.

Next let us discuss the case of $\Gamma_{i j}^{\phi}=0$, which is due to some symmetries imposed on the $i$ - and $j$-matter fields. The 2-body decay then becomes suppressed by the mass of the final-state particles, $M_{Q}$. From the Lagrangian (7), the effective interaction is given by

$$
\mathcal{L}=-\frac{1}{2} e^{K / 2}\left(K_{\phi} W_{i j}+W_{\phi i j}-2 \Gamma_{\phi i}^{k} W_{j k}\right) \phi \chi^{i} \chi^{j}+\text { h.c. },
$$

where we have assumed that $\left|D_{\phi} W\right| \ll|W|$. Here and in the followings, we assume that the matter fields are charged under some symmetries for simplicity. Then we can set $K_{i}, W_{i} \ll 1$ for the matter fields. It should be noticed that the second term in the bracket is necessary to ensure the Kähler invariance. For instance, if we apply the Kähler transformation, $K \rightarrow$ $K-\left\langle K_{\phi}\right\rangle \phi-\left\langle K_{\phi^{*}}\right\rangle \phi^{*}$, the first term vanishes and the second term compensates it. This becomes clear if we write the interactions in terms of $G$. The effective Lagrangian (12) is represented as

$$
\mathcal{L}=-\frac{1}{2} e^{G / 2}\left(G_{\phi i j}-2 \Gamma_{\phi i}^{k} G_{j k}\right) \phi \chi^{i} \chi^{j}+\text { h.c. },
$$

which is obviously invariant under the Kähler transformation. Note that $\Gamma_{\phi i}^{\ell}$ in Eq. (12) is different from $\Gamma_{i j}^{\phi}$ in Eq. (9). The coefficient, $\Gamma_{\phi i}^{k} \sim K_{\phi i k^{*}}$, can be nonzero easily. For instance, $\delta K \sim|\phi|^{2}|Q|^{2}$ leads to $\Gamma_{\phi Q}^{Q} \sim\langle\phi\rangle$, which is nonzero as long as $\langle\phi\rangle \neq 0$.

On the other hand, the 2-scalar production consists of the two channels; $\phi \rightarrow \phi^{i} \phi^{j}$ and $\phi \rightarrow \phi^{i} \phi^{* j}$. The former comes from the scalar potential;

$$
\mathcal{L}=-\frac{1}{2} e^{K}\left(K_{\phi} W_{i j}+W_{\phi i j}-2 \Gamma_{\phi i}^{k} W_{j k}\right)^{*} g^{\phi \phi^{*}} W_{\phi \phi} \phi \phi^{* i} \phi^{* j}+\text { h.c.. }
$$

We can easily check that this provides the same decay rate as that of the fermion final state induced by (12), i.e., $\Gamma\left(\phi \rightarrow \bar{\chi}^{i} \bar{\chi}^{j}\right)=\Gamma\left(\phi \rightarrow \phi^{i} \phi^{j}\right)$. Also the kinetic term of the scalar fields gives another decay channel, $\phi \rightarrow \phi^{i} \phi^{* j}$. However its amplitude is proportional to the scalar mass squared of the final state, noting $\left[\phi|Q|^{2}\right]_{D}=\phi\left(\partial^{2} Q^{*}\right) Q+\cdots$. Thus the process becomes dominant only when the scalar fields has a quite large soft scalar mass.

To summarize, the total decay rate of the 2-body final state from the interactions (12) and (14) is

$$
\begin{aligned}
\Gamma^{(2-\text { body })} & \equiv \Gamma\left(\phi \rightarrow \bar{\chi}^{i} \bar{\chi}^{j}\right)+\Gamma\left(\phi \rightarrow \phi^{i} \phi^{j}\right) \\
& \simeq \frac{C_{i j}^{(2)}}{8 \pi} m_{\phi}\left(1-\frac{4 M_{Q}^{2}}{m_{\phi}^{2}}\right)^{\frac{1}{2}}
\end{aligned}
$$



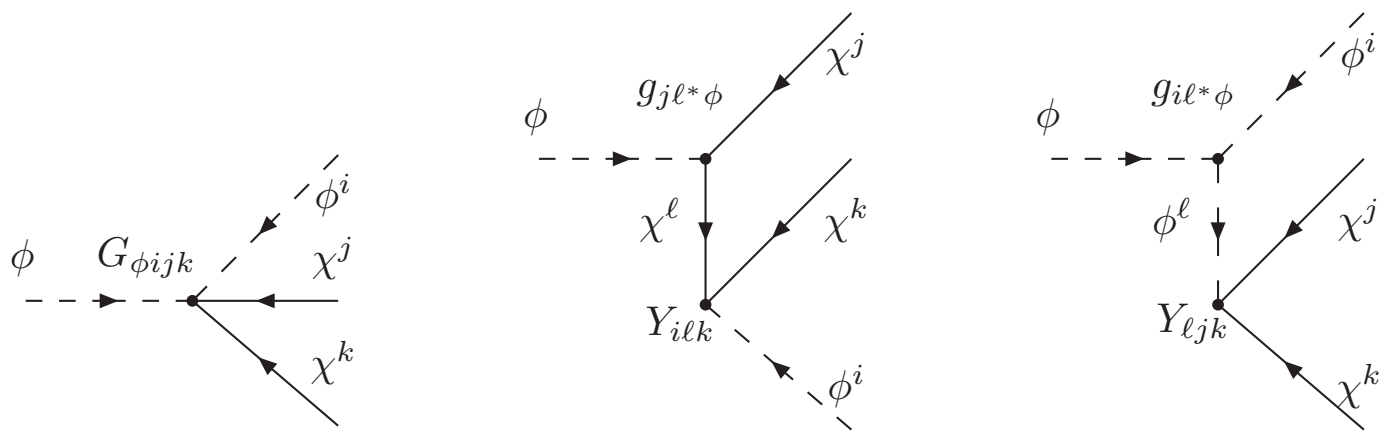

FIG. 1: The decay of the inflaton into the three-body final states; the decay with the four-point vertex, and with the fermion and scalar exchanges, from left to right.

where $C_{i j}^{(2)}=e^{K}\left|K_{\phi} W_{i j}+W_{\phi i j}-2 \Gamma_{\phi i}^{k} W_{j k}\right|^{2}$ with fixed $i$ and $j$ (the sum is taken only over $k$ ). If the particles in the final state have a SUSY mass, $W=M_{Q} Q Q^{\prime}, C_{i j}^{(2)}$ is proportional to $M_{Q}^{2}$. If the two particles in the final state are identical to each other, $W=\frac{1}{2} M_{Q} Q Q$ (e.g. the right-handed neutrino $N$ with a Majorana mass $M_{N}$ ), the decay rate becomes half of $(15)^{\mathrm{g}}$.

Next we consider the decay with 3-body final states. The decay processes through the dimension five operators are $\phi \rightarrow \phi^{* i} \bar{\chi}^{j} \bar{\chi}^{k}$ and $\phi \rightarrow \phi^{i} \phi^{j} \phi^{k}$. The former process is composed of the three diagrams in Fig. 1. In addition to the spontaneous decay process pointed out in [9] (the left diagram), the higher dimensional terms in the Kähler potential contribute to the decay rate (middle and right). Evaluating these diagrams, we obtain the effective interactions as

$$
\mathcal{L}=-\frac{1}{2} e^{K / 2}\left(K_{\phi} W_{i j k}+W_{\phi i j k}-3 \Gamma_{\phi i}^{\ell} W_{j k \ell}\right) \phi \phi^{i} \chi^{j} \chi^{k}+\text { h.c.. }
$$

On the other hand, the interactions representing the decay into 3 scalars, $\phi \rightarrow \phi^{i} \phi^{j} \phi^{k}$, are obtained by expanding the scalar potential as

$$
\mathcal{L}=-\frac{1}{6} e^{K}\left(K_{\phi} W_{i j k}+W_{\phi i j k}-3 \Gamma_{\phi i}^{\ell} W_{j k \ell}\right)^{*} g^{\phi \phi^{*}} W_{\phi \phi} \phi \phi^{* i} \phi^{* j} \phi^{* k}+\text { h.c.. }
$$

One can write down these interactions in terms of the Kähler invariant function, $G$, by replacing $K_{\phi} W_{i j k}+W_{\phi i j k} \rightarrow G_{\phi i j k}$ and $W_{j k \ell} \rightarrow G_{j k \ell}$, respectively. We find that the decay

\footnotetext{
$\mathrm{g}$ The spontaneous decay into the right-handed (s)neutrinos make the non-thermal leptogenesis scenario quite attractive [20].
} 
rate into 3 scalars is same as that into 1 scalar +2 fermions, i.e., $\Gamma\left(\phi \rightarrow \phi^{i} \phi^{j} \phi^{k}\right) \simeq \Gamma(\phi \rightarrow$ $\left.\phi^{* i} \bar{\chi}^{j} \bar{\chi}^{k}\right)+\Gamma\left(\phi \rightarrow \phi^{* j} \bar{\chi}^{k} \bar{\chi}^{i}\right)+\Gamma\left(\phi \rightarrow \phi^{* k} \bar{\chi}^{i} \bar{\chi}^{j}\right)$, for fixed $i, j$ and $k$. Summing these decay rates, the total 3 -body decay rate is given by

$$
\begin{aligned}
\Gamma^{(3-\text { body })} & \equiv \Gamma(\phi \rightarrow 3 \text { scalars })+\Gamma(\phi \rightarrow 1 \text { scalar }+2 \text { fermions }) \\
& \simeq \frac{C_{i j k}^{(3)}}{256 \pi^{3}} m_{\phi}^{3}
\end{aligned}
$$

where $C_{i j k}^{(3)}=e^{K}\left|K_{\phi} W_{i j k}+W_{\phi i j k}-3 \Gamma_{\phi i}^{\ell} W_{j k \ell}\right|^{2}$ with fixed $i, j$ and $k$ (sum over $\ell$ ). Here we have neglected the masses of the final-state particles.

Finally we discuss the inflaton decay into the gauge bosons and gauginos. At the tree level, it is effective only when the gauge kinetic function depends on the inflaton field ${ }^{\mathrm{h}}$. Actually, we obtain the total rate of the decay into the gauge sector as [4]

$$
\Gamma^{\text {(gauge tree) }} \simeq \frac{N_{g}}{4 \pi}|\kappa|^{2} m_{\phi}^{3},
$$

from $\mathcal{L}=\kappa \int d^{2} \theta \phi W^{\alpha} W_{\alpha}$, where $W_{\alpha}$ is a field strength of the gauge supermultiplet, $N_{g}$ is a number of the generators of the gauge symmetry, and we have assumed the canonical normalization for the inflaton and gauge multiplet. In (19), half of the decay rate comes from the gauge boson production and the other half is from the gaugino production.

Except for such direct couplings, no sizable interactions are found at the tree level between the inflaton and the gauge fields in the SUGRA Lagrangian [12]. The feature can be understood by using the gravity supermultiplet. The multiplet is minimally composed of the followings;

$$
h_{m n}, \quad \psi_{m}^{\alpha}, \quad b_{m}, \quad M
$$

which represent the graviton, the gravitino, and the vector and scalar auxiliary fields that correspond to the $U(1)_{R}$ and conformal symmetries of the superconformal transformation, respectively. Even in the absence of the direct couplings, the gravity multiplet can connect the inflaton field to the visible/hidden sectors. Actually, the auxiliary fields, $b_{m}$ and $M$, depend on the inflaton field as well as the visible/hidden fields due to the equation of motion, and the longitudinal component of the graviton, $h$, is related to the inflaton through the Lagrangian term, $\mathcal{L}=-\frac{1}{2} e^{-K / 3} \mathcal{R}$, in the conformal frame ${ }^{\mathrm{i}}$.

$\mathrm{h}$ The coupling may be induced by the mixing of the inflaton with other fields such as the SUSY breaking field [6].

i Even in the non-SUSY models, the latter contribution can arise [21]. 
The relevant terms involving $b_{m}$ in the SUGRA Lagrangian [12] are given by

$$
\mathcal{L}_{\text {aux }}=\frac{1}{3} b^{m} b_{m}-\frac{1}{3} i\left(K_{i} \partial_{m} \phi^{i}-K_{i^{*}} \partial_{m} \phi^{* i}\right) b^{m}+\frac{1}{6} g_{i j^{*}} \bar{\chi}^{j} \bar{\sigma}_{m} \chi^{i} b^{m}-\frac{1}{2} \bar{\lambda} \bar{\sigma}_{m} \lambda b^{m} .
$$

Solving the equation of motion for $b_{m}$, one can see that $b_{m}$ depends linearly on $\phi$ with a coefficient $K_{\phi}$, and that it also includes the gaugino current, $\bar{\lambda} \bar{\sigma}_{m} \lambda$. The decay into a pair of the gauginos is thus suppressed by the gaugino mass because the processes requires a chirality flip. In other words, noting that the inflaton contributes to the longitudinal component of $b_{m}$, the $U(1)_{R}$ charges of the final state should be nonzero for the decay to proceed due to the $U(1)_{R}$ current conservation. Thus the gaugino mass appears in the amplitude.

Next we focus on $h$ and $M$. The superconformal calculus formulation of SUGRA [22] is convenient to understand the decays mediated by these fields. In fact, their contributions can be taken into account by incorporating the chiral compensator field into the Lagrangian. The F-term of the compensator corresponds to $M$ by using the equation of motion, and $M$ includes a linear term with respect to $\phi$, whose coefficient is proportional to $K_{\phi}$. Further, since the compensator has a Weyl charge, its scalar component depends on $K$ after the Weyl transformation to canonicalize the gravity sector, i.e. from the conformal frame into the Einstein one. Then $\phi$ arises linearly in the scalar component when $K_{\phi}$ is non-zero. Thus the operators induced by $h$ and $M$ are represented by multiplying the compensator field. It is, however, known that the compensator does not physically couple to the gauge sector because it is conformal. Consequently, the decays into the gauge sector are suppressed at the tree level.

Before closing, it is interesting to note that these features are broken at the quantum level. That is, the inflaton can decay into the gauge sector via anomalies. We will discuss this mechanism in the next section.

\section{ANOMALY-INDUCED DECAY}

At the classical level, the spontaneous decay of the inflaton into the gauge sector is suppressed, since the gauge sector is conformal as discussed in the previous section. The quantum corrections, however, violate the conformal invariance, and so, the inflaton decay into the gauge sector may arise at the quantum level. Taking account of the SUGRA effects, the super-Weyl-Kähler (SW-Kähler) symmetry and the sigma-model isometry are 
anomalous at the quantum level. Not only do these anomalies mediate the SUSY-breaking effects to the visible sector [23], but they also enable the inflaton field to couple to the gauge supermultiplets [11].

In the superfield description, the 1PI effective action includes the non-local terms corresponding to the anomalies [24, 25];

$$
\begin{aligned}
\Delta \mathcal{L}=-\frac{g^{2}}{(16 \pi)^{2}} \int d^{2} \theta & W^{\alpha} W_{\alpha} \frac{\bar{D}^{2}}{\partial^{2}}\left[4\left(T_{R}-3 T_{G}\right) R^{\dagger}\right. \\
& \left.-\frac{T_{R}}{3} D^{2} K+\left.\frac{T_{R}}{d_{R}} D^{2} \log \operatorname{det} K\right|_{R} ^{\prime \prime}\right]+ \text { h.c. }
\end{aligned}
$$

at the leading order of $1 / M_{P}$ in the conformal frame. Here $D$ is a covariant derivative of the supersymmetry, and $g$ is a gauge coupling constant. The coefficients, $T_{G}$ and $T_{R}$, are the Dynkin index of the adjoint representation and matter fields in the representation $R$ of dimension $d_{R}$, which are normalized to $N$ for $\mathrm{SU}(N)$ and $1 / 2$ for its fundamentals. A sum over the matter fields is understood. Also $\left.K\right|_{R} ^{\prime \prime}$ denotes the Kähler metric restricted to the representation $R$. The first term in the bracket of Eq. (22) corresponds to the SW anomaly, and it is not invariant under the SW transformation. In fact, the superspace curvature $R$ changes under the SW transformation as [12];

$$
\delta R=-2(2 \Sigma-\bar{\Sigma}) R-\frac{1}{4} \bar{D}^{2} \bar{\Sigma}
$$

where a chiral superfield $\Sigma$ is defined so as to rescale the vielbein, $\delta E_{M}^{a}=(\Sigma+\bar{\Sigma}) E_{M}^{a}$, and the last term induces a shift of $R$. On the other hand, the second and third terms in Eq. (22) arise from the Kähler and sigma-model anomalies, respectively.

In the conformal frame, $R^{\dagger}$ is expanded as $R^{\dagger}=-\frac{1}{6}\left[M^{*}+\theta^{2}\left(-\mathcal{R} / 2+i \partial_{m} b^{m}\right)\right]+\cdots[12]$, where $\cdots$ is irrelevant for the decay. In addition to the auxiliary fields, the Ricci scalar, $\mathcal{R}$ depends on the inflaton field through the kinetic term, $\mathcal{L}=-\frac{1}{2} e^{-K / 3} \mathcal{R}$, which induces the mixing of the inflaton with the longitudinal mode of the graviton. To simplify the calculation, let us go to the Einstein frame where the gravity is canonically normalized. To this end, we perform the SW transformation with $\Sigma_{E}=\phi_{E}+\sqrt{2} \theta \chi_{E}+\theta^{2} F_{E}$ defined by [26]

$$
\phi_{E}=\frac{1}{12} K, \quad \chi_{E}=\frac{1}{6} K_{i} \chi^{i}, \quad F_{E}=\frac{1}{6} K_{i} F^{i}-\frac{1}{12} K_{i j} \chi^{i} \chi^{j} .
$$

Then the anomaly-induced term becomes [26]

$$
\Delta \mathcal{L}_{E}=\Delta \mathcal{L}+\frac{g^{2}}{16 \pi^{2}}\left(T_{R}-3 T_{G}\right) \int d^{2} \theta \Sigma_{E} W^{\alpha} W_{\alpha}+\text { h.c. },
$$


where the fields in $\Delta \mathcal{L}$ are simply replaced by those defined in the Einstein frame ${ }^{\mathrm{j}}$.

Expanding the superfields in terms of the components, one obtains interaction terms of the inflaton field to the gauge bosons/gauginos ${ }^{\mathrm{k}}$;

$$
\begin{aligned}
\mathcal{L} & =\frac{g^{2}}{64 \pi^{2}} X_{G} \phi\left(F_{m n} F^{m n}-i F_{m n} \tilde{F}^{m n}\right)-\frac{g^{2}}{32 \pi^{2}} X_{G} m_{\phi} \phi^{*} \lambda \lambda+\text { h.c. }, \\
X_{G} & =\left(T_{G}-T_{R}\right) K_{\phi}+\frac{2 T_{R}}{d_{R}}\left(\left.\log \operatorname{det} K\right|_{R} ^{\prime \prime}\right)_{, \phi}
\end{aligned}
$$

where $F_{m n}$ is a field strength of the gauge field and $\tilde{F}^{m n}=\epsilon^{m n k l} F_{k l} / 2$. Here we have also used the equations of motion for the auxiliary fields in the Einstein frame;

$$
b_{m}=\frac{1}{2} i\left(K_{i} \partial_{m} \phi^{i}-K_{i^{*}} \partial_{m} \phi^{* i}\right)+\cdots, \quad F^{i}=-e^{K / 2} g^{i j^{*}}\left(W_{j}+K_{j} W\right)^{*} .
$$

It is noticed that $M^{*}=-3 e^{K / 2} W^{*}$ does not induce the decay because of $\left|W_{\phi}\right| \sim m_{3 / 2}\langle\phi\rangle$ for the inflaton, $\phi$. The total decay rate from (26) becomes

$$
\Gamma^{\text {(anomaly })} \simeq \frac{N_{g} \alpha^{2}}{256 \pi^{3}}\left|X_{G}\right|^{2} m_{\phi}^{3}
$$

where $\alpha$ is a fine structure constant of the gauge group. Note that half of the decay rate comes from the decay into the two gauge bosons, while the other half from that into the gaugino pair.

Let us compare the rate of the anomaly-induced decay (28) with that of the spontaneous decay at the tree level (15) and (18). We find that all these rates are proportional to $\left|K_{\phi}\right|^{2}$. It means that, if the Kähler potential of the inflaton is canonical, the VEV of the inflaton field is necessary for the decay to proceed by the SUGRA effects. In contrast, the phase space and coupling constants depends on each process. The decay rate into the 2-body final state (15) is suppressed by the mass squared, $M_{Q}^{2} / M_{P}^{2} \ll 1$. While the rate of the 3-body final state (18) is suppressed by the phase space compared to (15). Instead, the coupling constant is given by the Yukawa coupling, $W_{i j k}$. Compared to these tree-level processes, the anomaly-induced decay takes place at the one-loop level. However, since the final state is 2 body, i.e. a pair of gauge bosons and gauginos, its rate is not negligible compared to those of the spontaneous decays at the tree level.

j A factor in front of $T_{R}$ is different from the result in [26] because here $K$ in $\Delta \mathcal{L}$ is not shifted.

k This result is also obtained at the component level by the Weyl rescaling, $e_{m}^{a} \rightarrow e^{-2 \sigma} e_{m}^{a}$, from the conformal frame to the Einstein frame. Then the $\mathcal{R}$ and $M$ shift as $\delta \mathcal{R}=12 \partial^{2} \sigma$ and $\delta M=-K_{i} F^{i}$ with $\sigma=K / 12$, while $b_{m}$ remains unchanged. 
Let us comment on a mass spectrum of the matters in the visible/hidden sectors. In this section, we have discussed anomalies that connects the inflaton with the gauge sector. In order for the process to occur, masses of the matters which contribute to the anomaly diagrams must be smaller than the inflaton mass. Otherwise the matters decouple from the anomalies. For instance, when we consider the anomaly-induced decay into the SUSY breaking sector, since masses in the hidden quarks are expected to be of $O(\Lambda)$, the decay takes place only for $m_{\phi}>\Lambda$.

So far, we have considered the anomalies of the SW-Kähler symmetry and sigma-model isometry. Since the process is an one-loop effect, there may be possible contributions from the counter term, depending on the underlying physics. Although we have assumed the conformal frame without the counter term at the cutoff scale in the above analyses, it can affect the decay rate, which is analogous to the anomaly-mediated SUSY breaking scenario [25].

Finally, let us comment on the inflaton decay into the SUSY breaking sector which involves the conformal dynamics. If the inflaton mass is above the scale of the violation of the conformal dynamics ${ }^{1}$, its decay into the SUSY breaking sector is expected to be suppressed. Actually, since the beta function vanishes above the scale, the decay induced by the SW anomaly is forbidden. At the same time, the contributions from the Kähler and sigma-model anomalies are implied to be suppressed ${ }^{\mathrm{m}}$, once we notice that the SUSY breaking sector is sequestered from the other sectors by the conformal dynamics [28]. Then the inflaton field may not decay into the conformal SUSY breaking sector, and so, the models will be free from the gravitino production.

\section{A. Minimal Kähler Potential}

Let us explicitly show several examples of the spontaneous and anomaly-induced decays. The former decay depends on the Kähler potential of the inflaton and visible/hidden sectors. Let us first discuss the case of the minimal Kähler potential. We take the Kähler potential

\footnotetext{
1 See [27] for a conformal theory of the SUSY breaking.
}

$m$ M.E. thanks K.-I. Izawa for discussions. 
and the superpotential as

$$
\begin{aligned}
K & =\phi \phi^{*}+Q Q^{*}, \\
W & =W(\phi)+\frac{1}{2} M Q Q+\frac{1}{6} Y_{i j k} Q^{i} Q^{j} Q^{k},
\end{aligned}
$$

where $Q$ denotes the visible/hidden matters ${ }^{\mathrm{n}}$. Then the total decay rate is the sum of the spontaneous and anomaly-induced decays, $\Gamma=\Gamma^{(\text {tree })}+\Gamma^{(\text {anomaly })}$. The former is given by

$$
\Gamma^{(\text {tree })} \simeq \frac{N^{(2)}}{16 \pi} \frac{\langle\phi\rangle^{2}}{M_{P}^{2}} \frac{M^{2}}{M_{P}^{2}} m_{\phi}\left(1-\frac{4 M^{2}}{m_{\phi}^{2}}\right)^{\frac{1}{2}}+\frac{N^{(3)}}{256 \pi^{3}} \frac{\langle\phi\rangle^{2}}{M_{P}^{2}}\left|Y_{i j k}\right|^{2} \frac{m_{\phi}^{3}}{M_{P}^{2}}
$$

for fixed $i, j$ and $k$ with $i \neq j \neq k$. Here $N^{(2)}$ and $N^{(3)}$ denote a number of the final states. On the other hand, the anomaly-induced decay depends on the gauge structure. The decay rate is

$$
\Gamma^{(\text {anomaly })} \simeq \frac{N_{g} \alpha^{2}}{256 \pi^{3}}\left(T_{G}-T_{R}\right)^{2} \frac{\langle\phi\rangle^{2}}{M_{P}^{2}} \frac{m_{\phi}^{3}}{M_{P}^{2}}
$$

In the above results, we have assumed that the inflaton mass is dominated by the SUSYinvariant mass term in the superpotential, and we have neglected the masses of the final states for the 3-body decay and the anomaly-induced decay.

\section{B. Sequestered Kähler Potential}

The next example is the Kähler potential with a sequestering form;

$$
K=-3 \log \left[1-\frac{1}{3}\left(\phi \phi^{*}+Q Q^{*}\right)\right] \text {, }
$$

with the superpotential (30). Noting $\left\langle\Gamma_{\phi i}^{k}\right\rangle=(\langle\phi\rangle / 3) \delta_{i}^{k}$, the rates of the spontaneous and anomaly-induced decays are

$$
\begin{aligned}
\Gamma^{\text {(tree })} & \simeq \frac{N^{(2)}}{144 \pi} \frac{\langle\phi\rangle^{2}}{M_{P}^{2}} \frac{M^{2}}{M_{P}^{2}} m_{\phi}\left(1-\frac{4 M^{2}}{m_{\phi}^{2}}\right)^{\frac{1}{2}} \\
\Gamma^{(\text {anomaly })} & \simeq \frac{N_{g} \alpha^{2} b_{0}^{2}}{2304 \pi^{3}} \frac{\langle\phi\rangle^{2}}{M_{P}^{2}} \frac{m_{\phi}^{3}}{M_{P}^{2}} .
\end{aligned}
$$

where $b_{0}$ is the beta function of the gauge symmetry, $b_{0}=3 T_{G}-T_{R}$. The tree-level decay arises via the mass term of $Q$, and the anomaly-induced decay is due to the SW anomaly,

$\mathrm{n}$ In addition, there may be a soft scalar mass in the Kähler potential. However it is irrelevant for the spontaneous and anomaly-induced decay processes. 
while the spontaneous decay via the Yukawa coupling vanishes. We also find that the radiative effects associated to the Kähler and sigma-model anomalies cancel with each other, which is analogous to the cancellation of the AMSB effects to the gaugino mass [23] .

The cancellation of the 3-body decay can be understood in the conformal frame. As was explained above, a part of the spontaneous decay is mediated by $b_{m}$ and the others are represented in terms of the chiral compensator field, $\Phi$. The auxiliary field $b_{m}$ behaves as the gauge field of $U(1)_{R}$ of the superconformal symmetry. At the tree-level, this symmetry is preserved by assigning an $U(1)_{R}$ charge $2 / 3$ for the chiral compensator. Then after a field redefinition, $\Phi Q \rightarrow Q, U(1)_{R}$ charge vanishes for the operators which represent the 3-body final state of the decay, that is, $\phi^{i} \chi^{j} \chi^{k}$ and $W_{\phi \phi} \phi^{i} \phi^{* j} \phi^{* k}$. Consequently, the decay mediated by $b_{m}$ cannot proceed via the Yukawa interaction. The other tree-level processes induced by the gravitational effects are also suppressed for the 3-body decay. They are obtained by multiplying $\Phi$. Remembering that the anomaly-mediated SUSY breaking contributions to the soft trilinear couplings are absent at the tree level, $\Phi$ does not contribute to the Yukawa interactions physically. Actually, $\Phi$ becomes absent in the Yukawa interaction by the redefinition of the matte field, $\Phi Q \rightarrow Q$.

In addition to the gravitational effects discussed above, the inflaton decay may be induced by higher dimensional operators in the Kähler function, $\Omega$, which is defined as $\Omega=-3 e^{-K / 3}$. In fact, in contrast to the sequestered Kähler potential, there exist the higher dimensional terms in $\Omega$ for the minimal Kähler potential. Thus the 3-body decays are allowed for the minimal case (see (31)), while they are absent in the sequestered one (see (34) $)^{\circ}$.

\section{COSMOLOGY}

We now consider cosmological implications of the inflaton decay processes discussed in the preceding sections. One immediate consequence is that the reheating temperature $T_{R}$ is bounded below; $T_{R}$ cannot be arbitrarily low, since the inflaton decays into the visible sector through the top Yukawa coupling (See Eq. (18)). The other is the gravitino production from inflaton decay, which can occur through three different processes: (i) gravitino pair

\footnotetext{
${ }^{\circ}$ In the Einstein frame, the cancellation can be seen explicitly by the field redefinition, $e^{\hat{K} / 6} Q \rightarrow Q$, with $\hat{K}=K-\langle K\rangle$. This rescaling substantially corresponds to the transformation from the Einstein frame to the conformal one with respect to the interaction terms of the matters.
} 
production; (ii) spontaneous decay at the tree level; (iii) anomaly-induced decay at the one-loop level. We will show how severely the gravitino production constrains the inflation models and SUSY breaking scenarios.

\section{A. Lower bound on the reheating temperature}

Let us begin with a relatively simple exercise. The supersymmetric SM sector contains the top Yukawa coupling in the superpotential as

$$
W=Y_{t} T Q H_{u}
$$

where $Y_{t}$ is the top Yukawa coupling, and $T, Q$, and $H_{u}$ are the chiral supermultiplets of the right-handed top quark and left-handed quark doublet of the third generation, and up-type Higgs, respectively. In this section, we assume that the inflaton has the minimal Kähler potential for simplicity. The partial decay rate of the inflaton through the top Yukawa coupling is then

$$
\Gamma_{T} \simeq \frac{3}{128 \pi^{3}}\left|Y_{t}\right|^{2}\langle\phi\rangle^{2} m_{\phi}^{3}
$$

where $\langle\phi\rangle$ and $m_{\phi}$ are VEV and the mass of the inflaton, respectively. The partial decay rate (36) is derived from Eq. (18) by noting $C^{(3)} \simeq Y_{t}^{2}|\langle\phi\rangle|^{2}$ and additional numerical factor 6 coming from $\mathrm{SU}(3) \times \mathrm{SU}(2)$. The presence of the decay through the top Yukawa coupling sets a lower bound on the reheating temperature, $T_{R}$. We define the reheating temperature as

$$
T_{R} \equiv\left(\frac{\pi^{2} g_{*}}{10}\right)^{-\frac{1}{4}} \sqrt{\Gamma_{\phi}}
$$

where $g_{*}$ counts the relativistic degrees of freedom, and $\Gamma_{\phi}$ denotes the total decay rate of the inflaton. Using Eqs. (36) and (37), we obtain the lower bound on $T_{R}$,

$$
T_{R} \gtrsim 1.9 \times 10^{3} \mathrm{GeV}\left|Y_{t}\right|\left(\frac{g_{*}}{200}\right)^{-\frac{1}{4}}\left(\frac{\langle\phi\rangle}{10^{15} \mathrm{GeV}}\right)\left(\frac{m_{\phi}}{10^{12} \mathrm{GeV}}\right)^{\frac{3}{2}} .
$$

Similarly the inflaton decays into the gluons and gluinos via the anomalies of SUGRA. One can estimate the decay rate from Eq. (28) as

$$
\Gamma_{\mathrm{SU}(3)} \simeq \frac{9}{32 \pi^{3}} \alpha_{s}^{2}\langle\phi\rangle^{2} m_{\phi}^{3}
$$

where $\alpha_{s}=g_{s}^{2} / 4 \pi$ denotes the $\mathrm{SU}(3)_{C}$ gauge coupling constant. Substituting $\alpha_{s} \simeq 0.05$, we can see $\Gamma_{\mathrm{SU}(3)}$ is one order of magnitude smaller than $\Gamma_{T}$. Therefore the spontaneous decay 
into the visible sector is dominated by that through the top Yukawa coupling, unless the Kähler potential takes a specific form, i.e. the sequestered type (see Sec. IVB).

We show the contours of the lower limit on $T_{R}$ given by Eq. (38) in Fig. 2, together with typical values of $\langle\phi\rangle$ and $m_{\phi}$ for the single-field new [29], multi-field new [30], hybrid [31] and smooth hybrid [32], and chaotic [33] inflation models. We will discuss each inflation model later in this section. If the inflaton mass $m_{\phi}$ and the $\mathrm{VEV}\langle\phi\rangle$ are too large, the reheating temperature may exceed the upper bound from the gravitinos produced by particle scattering in the thermal plasma. The cosmological constraints on the gravitino are summarized in Sec. VC. For more details, the reader should refer to Refs. [34, 35, 36] for the unstable gravitino, and Refs. [37, 38, 39, 40, 41, 42] for the stable one. For instance, the reheating temperature is necessarily higher than $10^{6} \mathrm{GeV}$ for the smooth hybrid inflation model, which is difficult to be reconciled with the gravitino of $m_{3 / 2}=O(0.1-1) \mathrm{TeV}[34]$ and $10 \mathrm{eV} \lesssim$ $m_{3 / 2} \lesssim 10 \mathrm{MeV}[37$ ].

It is remarkable that the inflaton decays into the visible sector once it acquires a finite VEV; we do not need to introduce any interactions between the inflaton and the SM sector by hand in the Einstein frame ${ }^{\mathrm{P}}$ in order to induce the reheating. On the other hand, it may pose a cosmological problem at the same time. If the hidden sector also has a Yukawa coupling or includes the SW-Kähler/sigma-model anomalies, unwanted relics such as the gravitino may be directly produced by the inflaton. We will focus on the issue in the rest of this section.

\section{B. Gravitino Production}

We consider the gravitino production from the inflaton decay. To make our analysis simple and conservative, we assume that the inflaton has the minimal Kähler potential and does not have any direct couplings with the SUSY breaking sector in the superpotential. If we introduce possible couplings between the inflaton and the SUSY breaking field, the gravitino overproduction problem generically becomes severer. We also assume the DSB scenario with the dynamical scale $\Lambda$. Then the SUSY breaking field $z$ usually has a scalar mass $m_{z}$ that is much larger than the gravitino mass. Although the precise value of $m_{z}$ is

\footnotetext{
$\mathrm{p}$ Note that the interpretation of higher dimensional operators depends on a choice of the frame of SUGRA.
} 


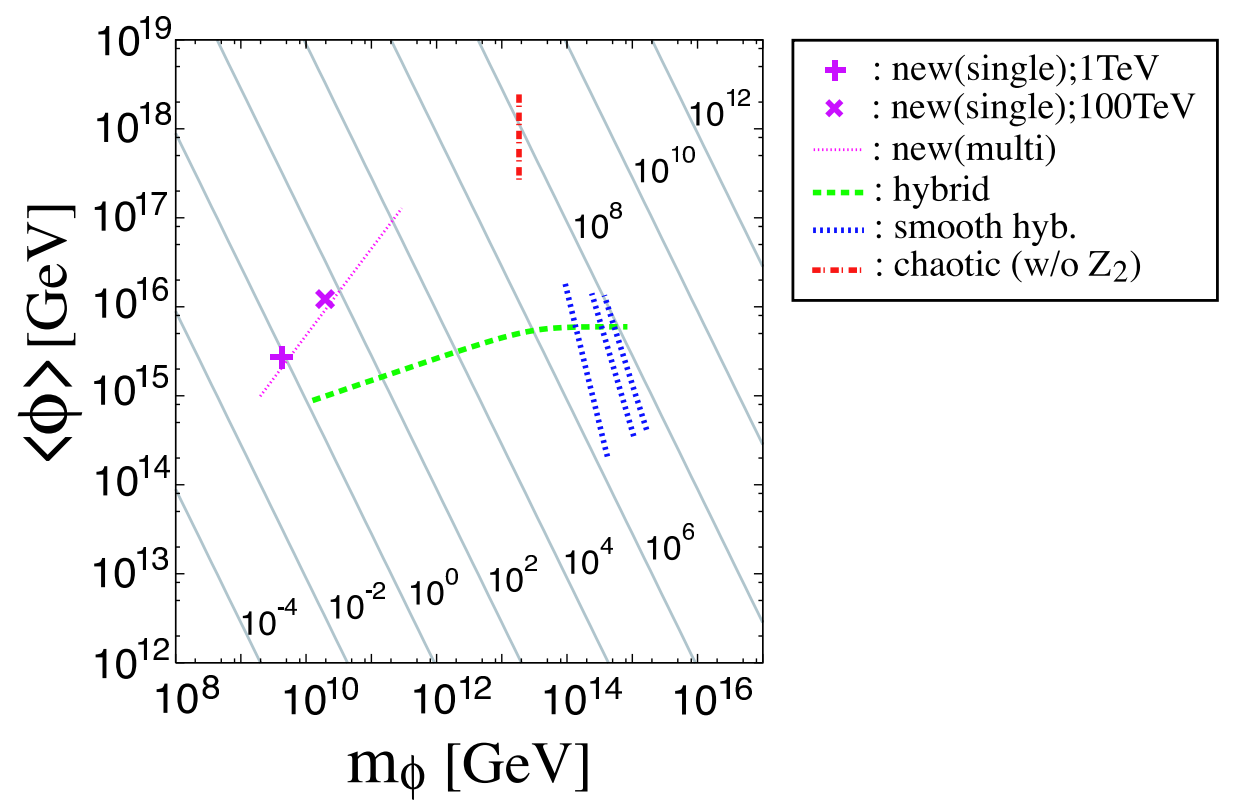

FIG. 2: Contours of the lower bound on $T_{R}$ in units of GeV. We set $g_{*}=228.75$ and $Y_{t}=0.6$. For details of the models, see Sec. VE,

model-dependent, it is expected to be of the order of $\Lambda$. Hereafter we simply assume ${ }^{\mathrm{q}}$

$$
m_{z} \simeq \Lambda \simeq \sqrt{m_{3 / 2}}
$$

We discuss the cases of $m_{\phi}>\Lambda$ and $m_{\phi}<\Lambda$ separately.

\section{The case of $m_{\phi}<\Lambda$}

As we have seen in Sec. II the inflaton decays into a pair of the gravitinos. The gravitino pair production is effective especially for a low-scale inflation model with $m_{\phi}<m_{z}$. The gravitino production rate is given by

$$
\Gamma_{3 / 2}^{(\text {pair })} \simeq \frac{1}{32 \pi}\langle\phi\rangle^{2} m_{\phi}^{3}
$$

for $m_{\phi}<m_{z} \simeq \Lambda$. Here we have assumed the minimal Kähler potential with a soft scalar mass of $z$ and $\langle z\rangle \ll 1$. The gravitino abundance is then

$$
Y_{3 / 2}=2 \frac{\Gamma_{3 / 2}^{(\text {pair })}}{\Gamma_{\phi}} \frac{3 T_{R}}{4 m_{\phi}}
$$

q The scalar mass $m_{z}$ can be smaller than $\Lambda$. If this is the case, the pair-gravitino production will be affected. 


$$
\simeq 7 \times 10^{-11}\left(\frac{g_{*}}{200}\right)^{-\frac{1}{2}}\left(\frac{T_{R}}{10^{6} \mathrm{GeV}}\right)^{-1}\left(\frac{\langle\phi\rangle}{10^{15} \mathrm{GeV}}\right)^{2}\left(\frac{m_{\phi}}{10^{12} \mathrm{GeV}}\right)^{2} .
$$

It should be noted that the gravitino abundance is inversely proportional to $T_{R}$. This feature is to be contrasted to the thermally produced gravitinos, whose abundance is proportional to $T_{R}$.

\section{The case of $m_{\phi}>\Lambda$}

When the inflaton mass $m_{\phi}$ is larger than $\Lambda$, the gravitational effects discussed in Sec. III and IV are important. If the SUSY breaking sector has Yukawa interactions, the inflaton decays into the sector via the operators. Besides, the anomalies of SUGRA induce the inflaton decay into the gauge boson and gauginos of the hidden gauge symmetries. Thus the hidden quarks and gauge bosons/gauginos are generally produced at the decay for $m_{\phi}>\Lambda$.

The hidden particles are energetic at the moment when they are produced. Since the reheating temperature $T_{R}$ is bounded as $T_{R}<\Lambda$ for almost entire region of the gravitino mass due to the thermal-gravitino production, the produced hidden particles do not reach thermal equilibrium. They instead form jets and hadronize by the strong gauge interactions, followed by cascade decays of the heavy hidden hadrons into lighter ones. The number of the hidden hadrons produced from each jet, which we call here as the multiplicity $N_{H}$, depends on the detailed structure of the hidden sector such as the gauge groups, the number of the matter multiplets, and a mass spectrum of the hidden hadrons. We expect $N_{H}$ to be in the range of $O\left(1-10^{2}\right)$.

The hidden hadrons should eventually decay and release their energy into the visible sector, since otherwise they will easily overclose the universe. The gravitinos are likely to be produced in the decays of the hidden hadrons as well as in the cascade decay processes in jets. This happens, e.g. through the kinetic mixings of the hidden matters, and especially if $z$ is a bound state of the hidden (s)quarks. Note that the goldstino is massless in the global SUSY limit and it is in the hidden sector with renormalizable couplings to other hidden (s)quark/gauge fields (and therefore hadrons). Thus, the goldstinos are expected to be produced by the hidden hadrons, though the precise production rate depends on details of the hidden sector. We denote the averaged number of the gravitinos produced per each jet as $N_{3 / 2}$. Here we assume each hidden hadron produces one gravitino in the end, and use 
the relation $N_{3 / 2} \sim N_{H}{ }^{\mathrm{r}}$.

The partial decay rates of the inflaton into the SUSY breaking sector are given by Eqs. (31) and (32). Although the DSB models do not always possess Yukawa interactions [43, 44, 45], all the DSB scenarios necessarily involve the gauge interactions. From Eq. (32), the partial rate of the inflaton decay into the SUSY breaking sector is:

$$
\Gamma_{\mathrm{DSB}}=\frac{N_{g}^{(h)} \alpha_{h}^{2}}{256 \pi^{3}}\left(T_{G}^{(h)}-T_{R}^{(h)}\right)^{2}\langle\phi\rangle^{2} m_{\phi}^{3}
$$

where the gauge coupling, the Dynkin indices, and the number of the generators are those of the hidden gauge symmetries. Multiplying the number of jets and $N_{3 / 2}$, the gravitino abundance becomes

$$
\begin{aligned}
Y_{3 / 2} & =2 N_{3 / 2} \frac{\Gamma_{\mathrm{DSB}}}{\Gamma_{\phi}} \frac{3 T_{R}}{4 m_{\phi}} \\
& \simeq 9 \times 10^{-13} \xi\left(\frac{g_{*}}{200}\right)^{-\frac{1}{2}}\left(\frac{T_{R}}{10^{6} \mathrm{GeV}}\right)^{-1}\left(\frac{\langle\phi\rangle}{10^{15} \mathrm{GeV}}\right)^{2}\left(\frac{m_{\phi}}{10^{12} \mathrm{GeV}}\right)^{2}
\end{aligned}
$$

where we have defined $\xi \equiv N_{3 / 2} N_{g}^{(h)} \alpha_{h}^{2}\left(T_{G}-T_{R}\right)^{2}$, which is roughly expected to be in the range of $O\left(10^{-2}\right)$ to $O(10)$.

In the following numerical analysis, we take the anomaly-induced decay as a source of the gravitino production channel for $m_{\phi}>\Lambda$. As one can see from Eqs. (31) and (32), the decay rate is roughly comparable to that of the spontaneous decay via the Yukawa coupling. Thus if one includes the tree-level decay into the analysis, the constraints become severer slightly, and the results in the followings do not change essentially.

\section{Cosmological Constraints on Gravitinos}

Before going further, here we briefly summarize the cosmological constraints on the gravitinos, which will be used to put constraints on the inflation models later.

There are tight constraints on the gravitino abundance from BBN if the gravitino is unstable [34, 35, 36] s, and from the dark matter (DM) abundance for the stable gravitino [37, 38, 39, 40, 41, 42]. The abundance of the gravitinos produced by thermal scatterings is

\footnotetext{
$\mathrm{r}$ In particular, if $z$ is an elementary field and has a Yukawa coupling, the inflaton necessarily produces at least one goldstino by the decay through the coupling

s For early works, see Refs. [46, 47, 48, 49, 50, 51, 52, 53].
} 
related to $T_{R}$ as $[34, \underline{39}]$

$$
\begin{aligned}
Y_{3 / 2}^{(t h)} & \simeq 1.9 \times 10^{-12}\left[1+\left(\frac{m_{\tilde{g}_{3}}^{2}}{3 m_{3 / 2}^{2}}\right)\right]\left(\frac{T_{\mathrm{R}}}{10^{10} \mathrm{GeV}}\right) \\
& \times\left[1+0.045 \ln \left(\frac{T_{\mathrm{R}}}{10^{10} \mathrm{GeV}}\right)\right]\left[1-0.028 \ln \left(\frac{T_{\mathrm{R}}}{10^{10} \mathrm{GeV}},\right)\right],
\end{aligned}
$$

where we have taken $N=3$ for QCD and $m_{\tilde{g}_{3}}$ is the gluino running mass evaluated at $T=T_{R}$. Since the gravitino abundance $Y_{3 / 2}^{(t h)}$ is roughly proportional to $T_{R}, T_{R}$ is bounded from above.

Here we simply quote the bounds on $Y_{3 / 2}$ and $T_{R}$ summarized in Ref. [7]. If the gravitino is light, it is likely the lightest SUSY particle (LSP) and therefore stable with the R-parity conservation. The bounds on $Y_{3 / 2}$ (and $T_{R}$ ) then come from the requirement that the gravitino abundance should not exceed the present DM abundance ${ }^{\mathrm{t}}$ :

$$
m_{3 / 2} Y_{3 / 2} \leq \frac{\rho_{c}}{s} \Omega_{\mathrm{DM}} \lesssim 4.4 \times 10^{-10} \mathrm{GeV}
$$

where $\rho_{c}$ is the critical density, and we used $\Omega_{\mathrm{DM}} h^{2} \lesssim 0.12$ at $95 \%$ C.L. [2] in the second inequality. The upper bound on $T_{R}$ can be obtained by substituting Eq. (46) into Eq. (45) as

$$
T_{R} \lesssim\left\{\begin{array}{ll}
O(100) \mathrm{GeV} & \text { for } m_{3 / 2} \simeq 10^{-2}-10^{2} \mathrm{keV} \\
8 \times 10^{7} \mathrm{GeV}\left(\frac{m_{\tilde{g}_{3}}}{300 \mathrm{GeV}}\right)^{-2}\left(\frac{m_{3 / 2}}{1 \mathrm{GeV}}\right) & \text { for } \quad m_{3 / 2} \simeq 10^{-4}-10^{2} \mathrm{GeV}
\end{array} .\right.
$$

Note that we have conservatively neglected the contribution from the decay of the nextto-lightest SUSY particle. In the following analysis, we assume that the gravitino with a mass lighter than $10^{2} \mathrm{GeV}$ is the LSP and stable. When the gravitino is as light as $m_{3 / 2} \sim O(10) \mathrm{eV}$ [55], there are no constraints on $T_{R}$, since the energy density of the gravitino would be too small even if the gravitino is thermalized. 35]:

On the other hand, if the gravitino is unstable, BBN puts severe constraints on $Y_{3 / 2}[34$,

$$
Y_{3 / 2} \lesssim\left\{\begin{array}{l}
1 \times 10^{-16}-6 \times 10^{-16} \text { for } m_{3 / 2} \simeq 0.1-0.2 \mathrm{TeV} \\
4 \times 10^{-17}-6 \times 10^{-16} \text { for } m_{3 / 2} \simeq 0.2-2 \mathrm{TeV} \\
7 \times 10^{-17}-2 \times 10^{-14} \text { for } m_{3 / 2} \simeq 2-10 \mathrm{TeV} \\
6 \times 10^{-13}-2 \times 10^{-12} \text { for } m_{3 / 2} \simeq 10-30 \mathrm{TeV}
\end{array} \quad\left(B_{h} \simeq 1\right)\right.
$$

\footnotetext{
t The gravitinos non-thermally produced by the inflaton decay can be a dominant component of DM, for certain values of the inflaton parameters [54].
} 


$$
Y_{3 / 2} \lesssim\left\{\begin{array}{l}
1 \times 10^{-16}-5 \times 10^{-14} \text { for } m_{3 / 2} \simeq 0.1-1 \mathrm{TeV} \\
2 \times 10^{-14}-5 \times 10^{-14} \text { for } m_{3 / 2} \simeq 1-3 \mathrm{TeV} \quad\left(B_{h} \simeq 10^{-3}\right) \\
3 \times 10^{-14}-2 \times 10^{-13} \text { for } m_{3 / 2} \simeq 3-10 \mathrm{TeV}
\end{array}\right.
$$

The corresponding upper bounds on $T_{R}$ are

$$
\begin{aligned}
& T_{R} \lesssim\left\{\begin{array}{ll}
(1-4) \times 10^{6} \mathrm{GeV} \quad \text { for } m_{3 / 2} \simeq 0.1-0.2 \mathrm{TeV} \\
3 \times 10^{5}-4 \times 10^{6} \mathrm{GeV} \text { for } m_{3 / 2} \simeq 0.2-2 \mathrm{TeV} \\
5 \times 10^{5}-1 \times 10^{8} \mathrm{GeV} \text { for } m_{3 / 2} \simeq 2-10 \mathrm{TeV} \\
(3-10) \times 10^{9} \mathrm{GeV} \quad \text { for } m_{3 / 2} \simeq 10-30 \mathrm{TeV}
\end{array} \quad\left(B_{h} \simeq 1\right),\right. \\
& T_{R} \lesssim \begin{cases}1 \times 10^{6}-3 \times 10^{8} \mathrm{GeV} \text { for } m_{3 / 2} \simeq 0.1-1 \mathrm{TeV} \\
(1-3) \times 10^{8} \mathrm{GeV} & \text { for } m_{3 / 2} \simeq 1-3 \mathrm{TeV} \quad\left(B_{h} \simeq 10^{-3}\right) . \\
2 \times 10^{8}-1 \times 10^{9} \mathrm{GeV} \text { for } m_{3 / 2} \simeq 3-10 \mathrm{TeV}\end{cases}
\end{aligned}
$$

For the heavy gravitino of mass $\gtrsim 30(10) \mathrm{TeV}$, no stringent constraints are obtained from BBN. However, another constraint comes from the abundance of the LSP produced by the gravitino decay. Since the gravitino life time is rather long, the produced LSPs will not annihilate with each other. Thus the upper bounds on $Y_{3 / 2}$ and $T_{R}$ read

$$
m_{\mathrm{LSP}} Y_{3 / 2} \lesssim 4.4 \times 10^{-10} \mathrm{GeV}
$$

and

$$
T_{R} \lesssim 2.5 \times 10^{10}\left(\frac{m_{\mathrm{LSP}}}{100 \mathrm{GeV}}\right)^{-1} \mathrm{GeV}
$$

where $m_{\mathrm{LSP}}$ denotes the mass of the LSP.

As is well-known, all the above constraints have been usually applied for the gravitinos from the thermal production. Since the gravitinos are also non-thermally produced in inflaton decay, we obtain further constraints on $T_{R}, m_{3 / 2},\langle\phi\rangle$ and $m_{\phi}$ by requiring the abundance of the non-thermally produced gravitinos (42) and (44) to satisfy (46), (48), (49), or (152). As we will see later, these new constraints drive (some part of) the high-scale inflation models and the gravity mediation into a corner.

\section{Constraints on Inflation Models and SUSY breaking}

Now we would like to derive constraints on the inflation and SUSY breaking models, using the non-thermal production of the gravitinos discussed above together with the thermal 
process.

In Fig. 3, we show the constraints on the inflaton mass and VEV for $m_{3 / 2}=1 \mathrm{GeV}, 1 \mathrm{TeV}$, and $100 \mathrm{TeV}$, together with typical values of the inflation models. We discuss each model in the next subsection. The region above each solid line is excluded. We find that in the case of $m_{3 / 2}=1 \mathrm{TeV}$ with $B_{h}=1$, all the inflation models shown in the figure are excluded. For the gravitino mass lighter or heavier than the weak scale, the constraints become relaxed. The inflaton mass and its VEV depend on the inflation models. Generically speaking, for larger $m_{\phi}$ and $\langle\phi\rangle$, the constraints become severer, simply because more gravitinos are produced by the inflaton decay (see (42) and (44)). On the other hand, if the inflaton is charged under some symmetries, its VEV becomes suppressed or even forbidden especially when the symmetry is exact at the vacuum. Then the bounds can be avoided for such inflation models. This is the case of the chaotic inflation model with a discrete symmetry (note that the chaotic inflation model shown in Fig. 3 is that without such a symmetry).

The solid lines which denote the constraint are jaggy at an intermediate value of $m_{\phi}$. This is because the dominant production channels of the gravitinos changes. In the right side, the gravitinos are produced by the spontaneous and anomaly-induced decays, while the inflaton directly decays into a pair of the gravitinos in the left side. Note that we have assumed (40) and $\xi=1$ for simplicity.

In Fig. 3, we have set $T_{R}$ to be the highest value allowed by the constraints. As mentioned before, the abundance of the non-thermally produced gravitinos is inversely proportional to $T_{R}$, which is different from that of the thermally produced one (see (45)). If $T_{R}$ takes a smaller value, the constraints becomes severer. Thus, the bounds shown in Fig. 3 are the most conservative ones. Note that one may have to introduce couplings of the inflaton with the SM particles to realize the highest allowed reheating temperature.

Instead, taking $T_{R}$ as a free parameter, we show the constraints on the $m_{\phi}-T_{R}$ plane, for $m_{3 / 2}=1 \mathrm{GeV}, 1 \mathrm{TeV}$, and $100 \mathrm{TeV}$ with a fixed $\langle\phi\rangle=10^{15} \mathrm{GeV}$ in Fig. 4, The reheating temperature is bounded from above due to the thermal production of the gravitino. It is remarkable that we have lower bounds on $T_{R}$ due to the non-thermal processes. In the figure, we incorporated the spontaneous decay via the top Yukawa interaction, which also provides a lower bound on $T_{R}$ (see (38) $)$. One can see that the lower bound on $T_{R}$ becomes severer for larger $m_{\phi}$.

In Fig. 5, we show constraints on the $m_{3 / 2}-m_{\phi}$ plane for several values of $\langle\phi\rangle=10^{12}, 10^{15}$, 


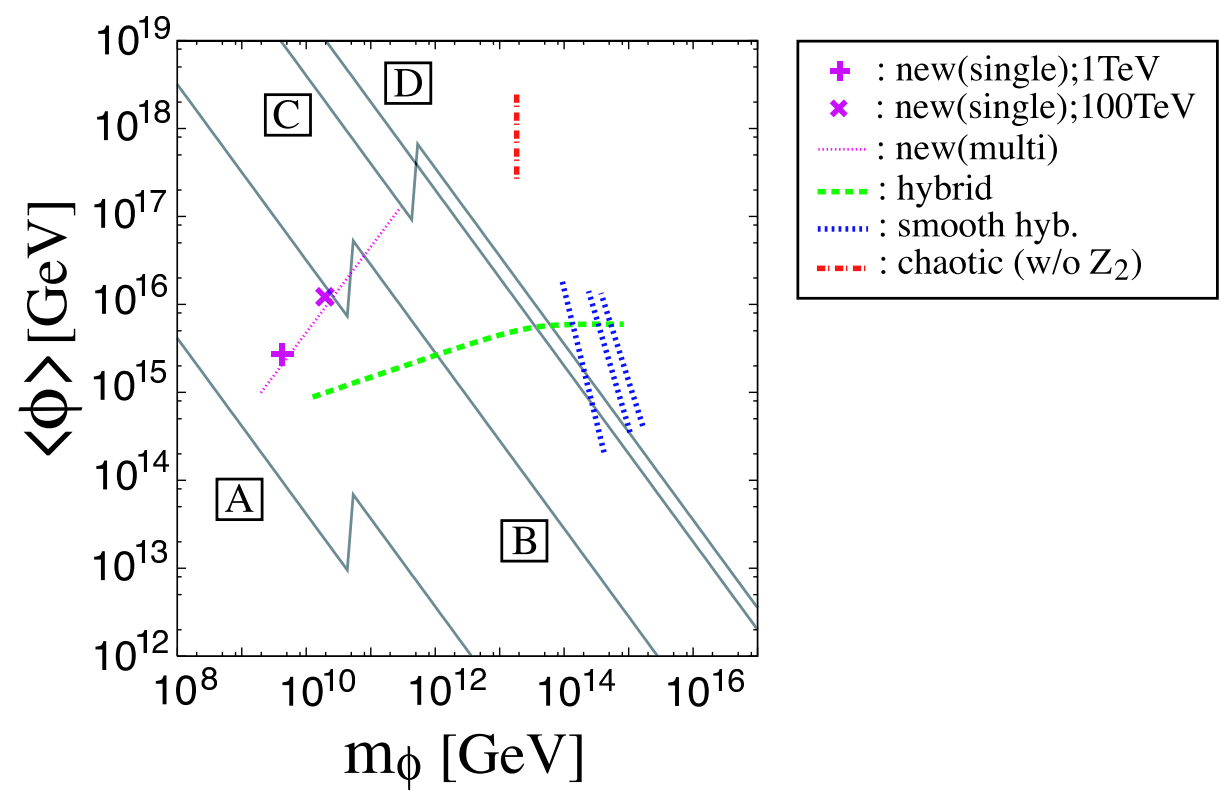

FIG. 3: Constraints from the gravitino production by the inflaton decay, for $m_{3 / 2}=1 \mathrm{TeV}$ with $B_{h}=1$ (case A), $m_{3 / 2}=1 \mathrm{TeV}$ with $B_{h}=10^{-3}$ (case B), $m_{3 / 2}=100 \mathrm{TeV}$ (case C), and $m_{3 / 2}=1 \mathrm{GeV}$ (case D). The region above the solid (gray) line is excluded for each case. For $m_{\phi} \gtrsim \Lambda$, we have used the anomaly-induced inflaton decay into the hidden gauge/gauginos to estimate the gravitino abundance, while the gravitino pair production has been used for $m_{\phi} \lesssim \Lambda$. Since $T_{R}$ is set to be the highest allowed value, the constraints shown in this figure are the most conservative ones.

and $10^{18} \mathrm{GeV}$. The dashed (pink) line represents $m_{\phi}=\Lambda$. For the inflaton mass $m_{\phi}$ above the dashed (pink) line, the spontaneous and anomaly-induced decays of the inflaton produce the gravitinos, while the pair production is dominant below the dashed (pink) line. We have set $T_{R}$ to be the highest value allowed by the constraints as we did in Fig. 3. We find that the inflaton mass cannot be too large, especially for $m_{3 / 2}$ around the weak scale. It is also noticed that the constraint becomes severer as $\langle\phi\rangle$ increases, since the upper bound on $m_{\phi}$ is proportional to $\langle\phi\rangle^{-1}$ for fixed $m_{3 / 2}$.

Finally let us illustrate how much the problem becomes severer in the case of the SUSY breaking models with an elementary singlet $z$. Such a field is needed to give sizable masses to the gauginos in the simple version of the gravity-mediation [14, 15] (see also footnote b). For an inflaton with $m_{\phi}<\Lambda$, the pair gravitino production occurs as described before. In particular, since $z$ is singlet at the cutoff scale, there is a priori no reason to forbid such 

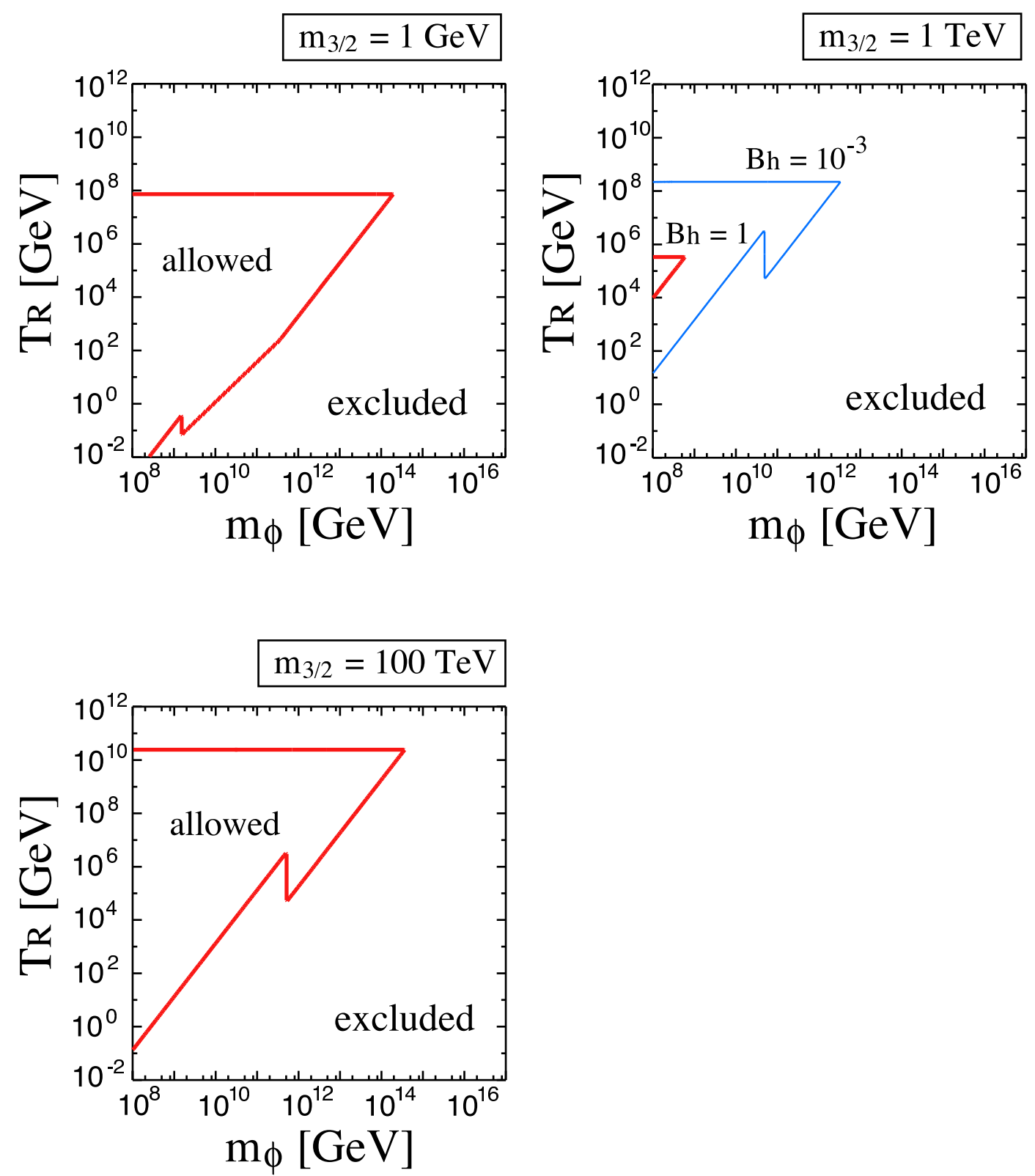

FIG. 4: Constraints from the gravitino production by the inflaton decay, for $m_{3 / 2}=1 \mathrm{GeV}$ (leftupper), $m_{3 / 2}=1 \mathrm{TeV}$ with $B_{h}=1$ and $10^{-3}$ (right-upper), $m_{3 / 2}=100 \mathrm{TeV}$ (bottom). We have set $\langle\phi\rangle=10^{15} \mathrm{GeV}$. The region surrounded by the solid line is allowed for each case.

an interaction as $\delta K \sim|\phi|^{2}\left(z+z^{*}\right)$. Then there generically exists a large kinetic mixing with the inflaton, and so, the gravitino production rate becomes too large, which is given by Eq. (6) with $\tilde{c} \sim 1$. With such a large gravitino production rate, most of the inflation models with $m_{\phi}<\Lambda$ are excluded, e.g. unless the inflaton VEV vanishes due to some 

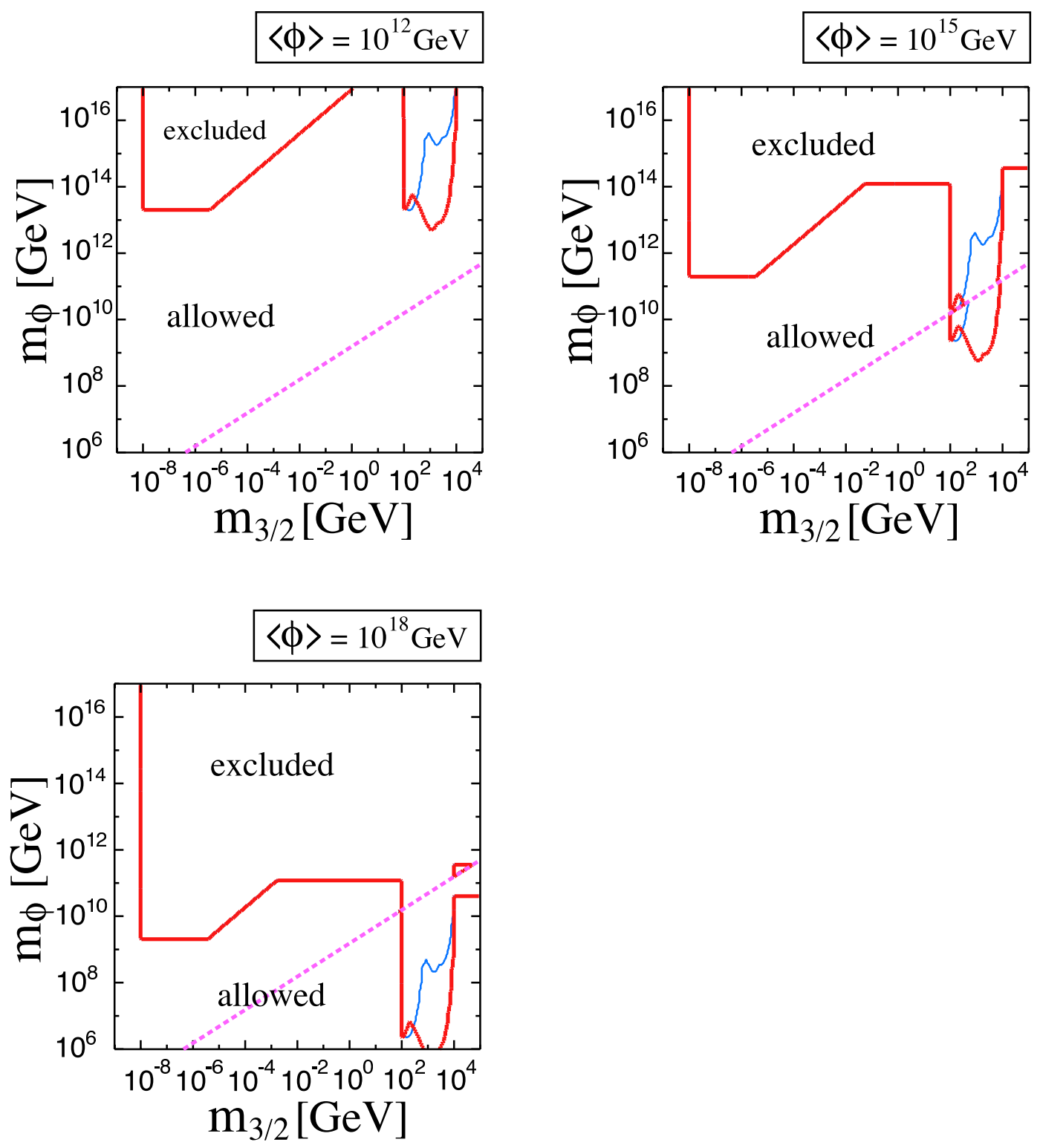

FIG. 5: Constraints from the gravitino production by the inflaton decay, for $\langle\phi\rangle=10^{12}, 10^{15}$ and $10^{18} \mathrm{GeV}$. The region above the thick solid line is excluded. We also show the constraint for the unstable gravitino with $B_{h}=10^{-3}$ as the thin (blue) line. For the region above the dashed (pink) line, we adopt (44), while (42) is used for the region below the dashed line. Since $T_{R}$ is set to be the highest allowed value, the constraints shown in this figure are the most conservative ones.

symmetries. Even for $m_{\phi}>\Lambda$, the pair gravitino production occurs effectively. In fact, one expects that $\delta K \sim|\phi|^{2} z z / 2+$ h.c. generally exists. The gravitino pair production is then given by (5) with $c \sim 1$. Thus the gravitino abundance increases by $O\left(10^{2}\right)$ compared to 
that from the spontaneous and anomaly-induced decays with $\xi=1$ for $m_{\phi}>\Lambda$ (see (42) and (44)) ${ }^{u}$. Besides, such a $z$ field may be displaced away from its potential minimum during the inflation, forcing the cosmological scenario to be more problematic (see the footnote C). Thus the SUSY breaking models with the elementary singlet $z$ are strongly disfavored from the cosmological points of view.

\section{E. Inflation Models}

In this subsection we give a brief review on the representative inflation models plotted in Figs. 2 and 3. For details on the models, the readers should refer to the original literatures.

\section{Single-field inflation model}

As a concrete example, here we study the new inflation model [29, 56, 57]. In the new inflation model, the Kähler potential and superpotential of the inflaton sector are written as $^{\mathrm{v}}$

$$
\begin{aligned}
K\left(\phi, \phi^{\dagger}\right) & =|\phi|^{2}+\frac{k}{4}|\phi|^{4} \\
W(\phi) & =v^{2} \phi-\frac{g}{n+1} \phi^{n+1}
\end{aligned}
$$

where the observed density fluctuations are explained for $v=4 \times 10^{-7}(0.1 / g)^{1 / 2}$ and $k \lesssim$ 0.03 in the case of $n=4$ [57]. After inflation, the inflaton $\phi$ takes the expectation value $\langle\phi\rangle \simeq\left(v^{2} / g\right)^{1 / n}$. In this model the inflaton mass is given by $m_{\phi} \simeq n v^{2} /\langle\phi\rangle$, and the gravitino mass is related to $v$ as $m_{3 / 2} \simeq n v^{2}\langle\phi\rangle /(n+1)$, since the inflaton induces the spontaneous breaking of the $R$-symmetry.

In the case of $n=4$, the inflaton parameters are $m_{\phi} \simeq 4 \times 10^{9} \mathrm{GeV}$ and $\langle\phi\rangle \simeq 3 \times 10^{15}$ $\mathrm{GeV}$ for $m_{3 / 2}=1 \mathrm{TeV}$, while $m_{\phi} \simeq 2 \times 10^{10} \mathrm{GeV}$ and $\langle\phi\rangle \simeq 1 \times 10^{16} \mathrm{GeV}$ for $m_{3 / 2}=100 \mathrm{TeV}$. Note that $m_{3 / 2} \ll 1 \mathrm{TeV}$ cannot be realized unless $g \gg 1$. From Fig. 3 , we can see that the new inflation model is excluded for $m_{3 / 2}=1 \mathrm{TeV}$ with $B_{h}=1$, while it is below the bound for $m_{3 / 2}=100 \mathrm{TeV}$.

\footnotetext{
u The spontaneous decay at the tree level and the anomaly-induced one into the SUSY breaking sector are not much affected by the presence of such an elementary singlet $z$.

$\mathrm{v}$ The gravitino abundance in the text remains virtually unchanged in the presence of the quartic coupling in the Kähler potential.
} 


\section{Multiple-field inflation model}

Next we consider an inflation model with multiple fields. Among many multiple-field inflation models proposed so far, there is an important class of models described by the following superpotential:

$$
W(\phi, \psi)=\phi f(\psi)
$$

where $f(\psi)$ is a function of $\psi$. The potential minimum in the global SUSY limit is located at

$$
\begin{aligned}
& \langle\phi\rangle=0, \\
& \langle\psi\rangle=\psi_{0},
\end{aligned}
$$

where $\psi_{0}$ satisfies $f\left(\psi_{0}\right)=0$. Note that the true minimum is slightly displaced from (56), once the SUSY breaking field is taken into account [7, 58].

For instance, the above class of the models includes a new inflation model [30] and a hybrid inflation model [31], described by

$$
W(\phi, \psi)=\phi\left(\mu^{2}-\frac{\psi^{n}}{M^{n-2}}\right),
$$

where $\mu$ determines the inflation energy scale and $M$ is an effective cut-off scale. In the new inflation model $\psi$ plays a role of the inflaton, while $\phi$ is the inflaton in the hybrid inflation model.

The inflaton fields $\phi$ and $\psi$ have almost the same masses,

$$
m_{\phi} \simeq m_{\psi} \simeq\left|e^{G / 2} \nabla_{\phi} G_{\psi}\right|
$$

which are assumed to be much larger than the gravitino mass. It should be noted that $\phi$ and $\psi^{\dagger}$ (and/or $\psi$ ) almost maximally mix with each other to form the mass eigenstates due to the almost degenerate masses [7]. To see this one should note that the difference between the diagonal components of the mass matrix is small: $\left|M_{\phi \bar{\phi}}^{2}-M_{\psi \bar{\psi}}^{2}\right|=O\left(m_{3 / 2}^{2}\right)$, while the off-diagonal component is relatively large: $M_{\phi \psi}^{2}=O\left(m_{3 / 2} m_{\phi}\right)$, resulting in the almost maximal mixing between $\phi$ and $\psi^{\dagger}$. Similar mixing may occur between $\phi$ and $\psi$. This mixing is effective at the inflaton decay, since the Hubble parameter at the decay should be smaller than $O\left(m_{3 / 2}\right)$ to satisfy the bounds from the thermally produced gravitinos. The 
mass eigenstates are obtained after taking account of the (almost) maximal mixing between $\phi$ and $\psi\left(\psi^{\dagger}\right)$ :

$$
\varphi_{ \pm} \simeq \frac{\phi \pm \psi^{(\dagger)}}{\sqrt{2}}
$$

The mass-eigenstates have the mass given by (58) and the effective $\mathrm{VEV}\left\langle\varphi_{ \pm}\right\rangle$given by $\psi_{0} / \sqrt{2}$ unless there is cancellation.

\section{2-A. New inflation model}

The new inflation discussed above is also realized for [30]

$$
\begin{aligned}
K & =|\phi|^{2}+|\psi|^{2}+\frac{k_{1}}{4}|\phi|^{4}+k_{2}|\phi|^{2}|\psi|^{2}+\frac{k_{3}}{4}|\psi|^{4}, \\
W & =\phi\left(v^{2}-g \psi^{4}\right),
\end{aligned}
$$

in which the inflaton is $\psi$, while $\phi$ stays at the origin during and after inflation. If one defines $k \equiv k_{2}-1$, the scalar potential for the inflaton $\psi$ becomes the same as the single-field new inflation model, although the gravitino mass is not related to the inflaton parameters. After the inflation ends, the energy of the universe is dominated by the oscillation energy of $\psi$. Although $\phi_{0}$ is suppressed compared to $\psi_{0}$, the effective VEV is given by $\psi_{0} / \sqrt{2}$, since $\phi$ and $\psi^{\dagger}$ almost maximally mixes with each other in the vacuum. Thus the constraint on this model is comparable to that on the single-field new inflation. We plot the values of $m_{\phi}$ and $\left\langle\varphi_{ \pm}\right\rangle$for $g=10^{-4}-1$ and $k=10^{-4}-10^{-1.5}$ with the e-folding number $N=50$ in Figs. 2 and 3. The (multi-field) new inflation model is excluded for $m_{3 / 2}=1 \mathrm{TeV}$ with $B_{h}=1$, while it is allowed for $m_{3 / 2}=1 \mathrm{GeV}$ and $100 \mathrm{TeV}$.

\section{2-B. Hybrid and Smooth hybrid inflation models}

The hybrid inflation model contains two kinds of superfields: one is $\phi$ which plays a role of inflaton and the others are waterfall fields $\psi$ and $\tilde{\psi}$ [31]. After inflation ends, $\phi$ as well as $\psi(\tilde{\psi})$ oscillates around the potential minimum and dominates the universe until the reheating.

The superpotential $W(\phi, \psi, \tilde{\psi})$ for the inflaton sector is

$$
W(\phi, \psi, \tilde{\psi})=\phi\left(\mu^{2}-\lambda \tilde{\psi} \psi\right)
$$


where $\psi$ and $\tilde{\psi}$ are assumed to be charged under $U(1)$ gauge symmetry. Here $\lambda$ is a coupling constant and $\mu$ is the inflation energy scale. The potential minimum is located at $\langle\phi\rangle=0$ and $\langle\psi\rangle=\langle\tilde{\psi}\rangle=\mu / \sqrt{\lambda}$ in the SUSY limit. For a successful inflation, $\mu$ and $\lambda$ are related as $\mu \simeq 2 \times 10^{-3} \lambda^{1 / 2}$ for $\lambda \gtrsim 10^{-3}$, and $\mu \simeq 2 \times 10^{-2} \lambda^{5 / 6}$ for $\lambda \lesssim 10^{-3}$.

Due to the D-term potential one linear combination of $\psi$ and $\tilde{\psi}$, given by $\psi^{(-)} \equiv(\psi-$ $\tilde{\psi}) / \sqrt{2}$, has a large mass of $\sim g\langle\psi\rangle$ ( $g$ denotes the gauge coupling), while the other, $\psi^{(+)} \equiv$ $(\psi+\tilde{\psi}) / \sqrt{2}$ has a mass equal to that of $\phi: m_{\psi^{(+)}}=m_{\phi}=\sqrt{2} \lambda\langle\psi\rangle$. It is the latter that (almost) maximally mixes with $\phi$ to form mass eigenstates. Note that $\mathrm{VEV}$ of $\psi^{(+)}$is equal to $\sqrt{2}\langle\psi\rangle$.

For $\lambda \sim 10^{-1}-10^{-5}$ [59] we obtain $\mu \sim 8 \times 10^{-4}-1 \times 10^{-6}, m_{\phi} \sim 10^{15}-10^{10} \mathrm{GeV}$, and $\left\langle\varphi_{ \pm}\right\rangle=\mu / \sqrt{\lambda} \sim O\left(10^{15}\right) \mathrm{GeV}$. From Fig. 3, one can see the hybrid inflation model is excluded by the gravitino overproduction for $m_{3 / 2}=1 \mathrm{TeV}$ with $B_{3 / 2}=1$. For $m_{3 / 2}=1 \mathrm{GeV}$ and $100 \mathrm{TeV}$, the constraints become slightly mild, but a certain fraction of the parameter space is still excluded. The allowed parameter space corresponds to $\lambda \lesssim 10^{-2}$. Note that the parameter space allowed by the gravitino production leads to almost scale-invariant power spectrum, which is disfavored by the WMAP data [2]. It is possible to make the scalar spectral index $n_{s}$ smaller than 1 by introducing non-renormalizable interactions in the Kähler potential [59, 60].

Here we comment on interesting observation concerning the spectral index and the cosmic string. In this type of hybrid inflation, cosmic strings are formed after inflation because $\psi$ and $\tilde{\psi}$ have $U(1)$ gauge charges. As is well known, the cosmic strings contribute to the density fluctuations. Including the effects of the cosmic string makes the spectral index $n_{s}$ between 0.98 and 1 compatible with the WMAP data [61, 62], if the tension of the cosmic string is $G \mu=O\left(10^{-7}\right)$. According to Ref. [61], this corresponds to the region with $\lambda \sim O\left(10^{-3}-10^{-2}\right)$. Interestingly enough, the region is just below the constraints from the gravitino production in the case of $m_{3 / 2}=1 \mathrm{GeV}$ and $100 \mathrm{TeV}{ }^{\mathrm{w}}$. This means that, for that region, the gravitino non-thermally produced by the inflaton decay may account for the present DM abundance [54], if the gravitino is stable. For the unstable gravitino of a mass $m_{3 / 2} \gtrsim O(10) \mathrm{TeV}$, Wino-like LSP produced by the gravitino decay may be the

\footnotetext{
w Including the soft terms, the inflaton dynamics is somewhat modified, and correspondingly the inflaton parameters are slightly changed, especially if the gravitino mass is heavy [63].
} 
dominant component of DM. Moreover, since the required tension of the cosmic string is relatively large and is close to the present observational upper bound, one may be able to discover the cosmic string in the future observations. Since the inflaton mass and the VEV are small, it is difficult to realize the non-thermal leptogenesis via the spontaneous decay (see Eq. (15)). However, one can naturally incorporate the non-thermal leptogenesis into the hybrid inflation model by identifying the $U(1)$ symmetry with a $U(1)_{B-L}$ symmetry [64].

Next let us consider a smooth hybrid inflation model [32], which predicts the scalar spectral index as $n_{s} \simeq 0.97$, which is slightly smaller than the simple hybrid inflation model. The superpotential of the inflaton sector is

$$
W(\phi, \psi, \tilde{\psi})=\phi\left(\mu^{2}-\frac{(\tilde{\psi} \psi)^{n}}{M^{2 n-2}}\right) .
$$

The VEVs of $\psi$ and $\tilde{\psi}$ are given by $\langle\psi\rangle=\langle\tilde{\psi}\rangle=\left(\mu M^{n-1}\right)^{1 / n}$, and we assume that $\psi=\tilde{\psi}$ always holds due to the additional D-term potential. Then one of the combination, $\psi^{(+)} \equiv$ $(\psi+\tilde{\psi}) / \sqrt{2}$, almost maximally mixes with $\phi$ to form the mass eigenstate of a mass $m_{\phi}=$ $\sqrt{2} n \mu^{2} /\langle\psi\rangle$. For $n=2$ we obtain $\mu \sim 4 \times 10^{-4}-9 \times 10^{-5}$, and $m_{\phi} \sim 1 \times 10^{14}-6 \times 10^{14} \mathrm{GeV}$. From Fig. 3, one can see that the smooth hybrid inflation model is excluded for a broad range of $m_{3 / 2}$.

\section{2-C. Chaotic inflation model}

A chaotic inflation [65] is realized in SUGRA, based on a Nambu-Goldstone-like shift symmetry of the inflaton chiral multiplet $\phi$ [33]. Namely, we assume that the Kähler potential $K\left(\phi, \phi^{\dagger}\right)$ is invariant under the shift of $\phi$,

$$
\phi \rightarrow \phi+i A
$$

where $A$ is a dimensionless real parameter. Thus, the Kähler potential is a function of $\phi+\phi^{\dagger}$; $K\left(\phi, \phi^{\dagger}\right)=K\left(\phi+\phi^{\dagger}\right)=c\left(\phi+\phi^{\dagger}\right)+\frac{1}{2}\left(\phi+\phi^{\dagger}\right)^{2}+\cdots$, where $c$ is a real constant and must be smaller than $O(1)$ for a successful inflation. As opposed to the other inflation models, this model allows a linear term in the Kähler potential. The coefficient $c$ corresponds to the inflaton $\mathrm{VEV}$ in the other models. If there is no other symmetry such as a $Z_{2}$ symmetry, there is no reason to expect that $c$ is much smaller than unity. 
We identify the imaginary part of $\phi$ with the inflaton field $\varphi \equiv \sqrt{2} \operatorname{Im}[\phi]$. Moreover, we introduce a small breaking term of the shift symmetry in the superpotential in order for the inflaton $\varphi$ to have a potential:

$$
W(\phi, \psi)=m \phi \psi
$$

where we introduced a new chiral multiplet $\psi$, and $m \simeq 2 \times 10^{13} \mathrm{GeV}$ determines the inflaton mass.

One might suspect that it is only the real component of $\phi$ that can decay into the gravitinos, since the shift symmetry dictates that the only real component $\left(\phi+\phi^{\dagger}\right)$ appears in the Kähler potential. However, it is not surprising that this is not the case, since the decay amplitude is proportional to powers of the large SUSY mass $m$ that explicitly violates the shift symmetry.

We plot the chaotic inflation model with $c=0.1-1$ in Figs. 2 and 3. One can see that it is excluded for almost entire values of $m_{3 / 2}$ (except for $m_{3 / 2} \lesssim O(10) \mathrm{eV}$ ). Note however that one can avoid the constraints by assuming an approximate $Z_{2}$ symmetry to suppress $c$.

\section{F. Possible solutions to the gravitino problem}

Here let us briefly mention possible solutions to the gravitino overproduction problem. As mentioned above, one solution is to postulate a symmetry of the inflaton. If the symmetry is unbroken at the vacuum (or if the breaking of this symmetry is small), the VEV of the inflaton, $\langle\phi\rangle$, is zero (or suppressed). As the gravitino production rate is proportional to $\langle\phi\rangle^{2}$, one can avoid the gravitino overproduction for such inflation models. Note however that, if the symmetry is exact, the visible matter fields as well must be charged under the same symmetry, since otherwise the inflaton cannot decay into the visible sector. This solution can be achieved e.g. in the chaotic inflation model; one can assign $Z_{2}$ symmetry on the inflaton [20]. Also, there are inflation models in which the inflaton is identified with the MSSM fields [66, 67, 68, 69] or the right-handed sneutrino [70]. By similar reasoning, the gravitino overproduction from the inflaton decay can be avoided in these models.

So far, we have assumed that there is no late-time entropy production after the inflaton decay. If huge entropy production [71, 72] occurs after the reheating of the inflaton, any preexisting gravitinos are diluted. However, since it also dilutes the pre-existing baryon number, one may have to generate the baryon asymmetry after the entropy production [73, 74, 75, 76]. 
Note also that the decay processes discussed in this paper can be applied to any scalar fields, and so, the scalar field that induces the large entropy may produce the gravitinos again. One has to make sure that this does not happen.

Another solution is to assume that the gravitino mass is either extremely heavy or extremely light. If the gravitino mass exceeds $O\left(10^{6}\right) \mathrm{GeV}$, the gravitino may decay before the decoupling of the LSP particle. To realize this, however, one has to contrive a set-up in which anomaly-mediation is suppressed. If we stick to the gravitino mass smaller than $100 \mathrm{TeV}$ on the basis of naturalness, the gravitino problem sets severe bounds on the highscale inflation models as seen above. On the other hand, if the gravitino mass is lighter than $O(10) \mathrm{eV}$, the gravitino is thermalized and its cosmological abundance is negligibly small [55]. So, the gravitino problem is absent for such a very light gravitino. Note that, in order to ameliorate the gravitino problem instead of solving it completely, one does not have to go to such extremes. For the gravitino mass moderately lighter or heavier than the weak scale, the gravitino problem is relaxed especially for the low-scale inflation models (see Fig. 3).

If the inflaton mass is quite light, one can evade the constraints as one can see from Fig. 3 , However, it should be noted that the reheating temperature is set to be the highest allowed value in Fig. 3, For a lower reheating temperature, the constraints become severer. This means that, one may have to introduce relatively strong couplings of the inflaton to the SM particles in order to realize the reheating temperature adopted in Fig. 3, For instance, if the inflaton has only the dimension-five couplings to the visible sector suppressed by the Planck scale, the reheating temperature $T_{R}$ is proportional to $m_{\phi}^{3 / 2}$, which makes the gravitino abundances (42) and (44) rather insensitive to the inflaton mass. Then, one cannot evade the gravitino problem simply by changing the inflaton mass ${ }^{\mathrm{x}}$.

Lastly, let us comment on the SUSY breaking sector which involves the conformal dynamics. As mentioned in Sec. IV] the inflaton field with $m_{\phi} \gg \Lambda$ may not decay into the conformal SUSY breaking sector. Then the gravitino production is suppressed. This solution is appealing because one does not have to impose non-trivial constraints on the inflation models or on the thermal history of the universe. The only requisite is the conformal dynamics in the SUSY breaking sector, which has its own phenomenological virtues

\footnotetext{
$\mathrm{x}$ This is one of the reasons why the moduli-induced gravitino problem [4] is quite difficult to be solved.
} 
independently of the gravitino overproduction problem. Furthermore, it may naturally lead to the successful non-thermal leptogenesis scenario, which will be discussed elsewhere [77].

\section{G. Comments on preheating}

Here we would like to mention the effects of the preheating [78], i.e., the non-perturbative inflaton decay process, which we have not taken into account so far. The non-thermal gravitino production from the inflaton decay has somewhat checkered history; it was once claimed that the gravitinos were non-thermally produced during preheating [79], but it was later concluded that the inflatino, instead of the gravitino in the low energy, was actually created [80]. Since the inflatino decays much earlier than the BBN epoch [81], the nonthermal 'gravitino' (actually, inflatino) production turned out to be harmless. However, as discussed so far, we have found that the gravitinos are generically produced by the perturbative decay processes.

Another concern is whether our results are modified by including the effects of the preheating [82]. We believe that our arguments on the gravitino overproduction problem are robust and they are not essentially modified even if the preheating occurs. First of all, we would like to emphasize that the decay processes discussed so far are perturbative ones, and therefore they are always present. On the other hand, it crucially depends both on the global structure of the inflaton potential and on the couplings of the inflaton to matter fields whether the preheating occurs and how efficiently it proceeds.

Let us assume that the preheating actually occurs and it proceeds quite efficiently without any back reaction, i.e., the inflaton transfers most of its energy into other particles soon after the inflaton starts oscillating. This corresponds to the instantaneous reheating, which generically leads to the overproduction of the gravitinos due to particle scatterings, instead of the non-thermal production. The latter is suppressed in this case since the reheating temperature will become high (see (42) and (44)). Therefore, for the most inflation models, such an efficient preheating should not occur, since otherwise too many gravitinos are produced by the conventional thermal scatterings.

On the other hand, if the preheating is not so efficient, then one has to take account of the back reaction processes, and the preheating typically ends at a certain point. The reheating of the universe is induced by the perturbative decay of the inflaton in the end, 
and the gravitinos are generically produced by the decay. Thus it is unlikely that the preheating solves or ameliorates the gravitino problem; one has to contrive a model in which the preheating proceeds quite efficiently, but the gravitinos are not produced by the scatterings of the decay products. Specifically, the decay products should not reach thermal equilibrium.

\section{CONCLUSION}

The present observational data on the CMB and the large-scale structure, together with the strong theoretical motivation to resolve severe problems in the standard big bang cosmology, have led us to believe that the universe underwent an inflationary epoch at an early stage. While there are many inflation models (called as "the inflationary zoo"), we still do not know which inflation model is realized in nature. The study on the density fluctuations such as isocurvature perturbations, non-gaussianity, tensor-mode, and their effects on the CMB power spectrum is quite useful, but is not enough at present to pin down the inflation model. This is partly because of our ignorance of thermal history of the universe beyond the standard big bang theory, e.g., how the inflaton reheats the universe.

In this paper, we have investigated the inflaton decay processes in the supergravity. In particular, we have shown that the gravitinos are generically produced in the inflaton decay. There are three different processes for the production. One is the direct production of a pair of the gravitinos. This is effective especially for low-scale inflation models. The other two are due to the inflaton decay into the SUSY breaking sector; the spontaneous decay at the tree level and the anomaly-induced one at the one-loop level. Those non-thermally produced gravitinos set tight constraints on the inflation models, together with the constraints from thermally produced gravitinos. Indeed, these two constraints are complementary in a sense that the dependence on the reheating temperature is different. For higher $T_{R}$, more gravitinos are thermally produced, while the non-thermally production becomes important for lower $T_{R}$. They also depend on the SUSY breaking scenarios. In fact, almost all parameter space for the inflaton is excluded for the gravity-mediated SUSY breaking scenarios.

Apart from the gravitino productions, the inflaton naturally decays into the visible sector especially through the top Yukawa coupling and $\mathrm{SU}(3)_{C}$ gauge interaction. Thus one does not need to introduce any direct ad hoc couplings by hand in order to induce the reheating. 
The above studies may provide us with a breakthrough toward the full understanding of the inflationary universe. In addition to the standard analysis on the density fluctuations, the inflation models in supergravity are subject to the constraints due to the (non)-thermally produced gravitinos. Whether a consistent thermal history after inflation is realized now becomes a new guideline to sort out the inflationary zoo, and hopefully it will pin down the true model, together with data in the future collider experiments such as LHC.

[1] A. H. Guth, Phys. Rev. D 23, 347 (1981); A. D. Linde, Phys. Lett. B 108, 389 (1982);

A. Albrecht and P. J. Steinhardt, Phys. Rev. Lett. 48, 1220 (1982).

[2] D. N. Spergel et al., arXiv:astro-ph/0603449.

[3] K. Sato, Mon. Not. Roy. Astron. Soc. 195, 467 (1981).

[4] M. Endo, K. Hamaguchi and F. Takahashi, Phys. Rev. Lett. 96, 211301 (2006);

S. Nakamura and M. Yamaguchi, Phys. Lett. B 638, 389 (2006).

[5] M. Dine, R. Kitano, A. Morisse and Y. Shirman, Phys. Rev. D 73, 123518 (2006).

[6] M. Endo, K. Hamaguchi and F. Takahashi, Phys. Rev. D 74, 023531 (2006).

[7] M. Kawasaki, F. Takahashi and T. T. Yanagida, Phys. Lett. B 638, 8 (2006); Phys. Rev. D 74, 043519 (2006).

[8] T. Asaka, S. Nakamura and M. Yamaguchi, Phys. Rev. D 74, 023520 (2006).

[9] M. Endo, M. Kawasaki, F. Takahashi and T. T. Yanagida, Phys. Lett. B 642, 518 (2006).

[10] M. Endo, K. Kadota, K. A. Olive, F. Takahashi and T. T. Yanagida, JCAP 0702, 018 (2007).

[11] M. Endo, F. Takahashi and T. T. Yanagida, arXiv:hep-ph/0701042.

[12] J. Wess and J. Bagger, Supersymmetry and Supergravity, (Princeton Unversity Press, 1992).

[13] E. Witten, Nucl. Phys. B 188, 513 (1981).

[14] G. D. Coughlan et al Phys. Lett. B 131 (1983) 59; B. de Carlos et al Phys. Lett. B 318 (1993) 447.

[15] T. Banks, D. B. Kaplan and A. E. Nelson, Phys. Rev. D 49, 779 (1994);

K. I. Izawa and T. Yanagida, Prog. Theor. Phys. 94, 1105 (1995);

A. E. Nelson, Phys. Lett. B 369, 277 (1996).

[16] M. Endo, F. Takahashi and T. T. Yanagida, arXiv:hep-ph/0702247.

[17] M. Ibe, Y. Shinbara and T. T. Yanagida, Phys. Lett. B 639, 534 (2006). 
[18] S. Weinberg, Phys. Rev. Lett. 48, 1303 (1982).

[19] L. M. Krauss, Nucl. Phys. B 227, 556 (1983).

[20] M. Endo, F. Takahashi and T. T. Yanagida, Phys. Rev. D 74, 123523 (2006).

[21] Y. Watanabe and E. Komatsu, Phys. Rev. D 75, 061301 (2007).

[22] E. Cremmer, B. Julia, J. Scherk, S. Ferrara, L. Girardello and P. van Nieuwenhuizen, Nucl. Phys. B 147, 105 (1979). E. Cremmer, S. Ferrara, L. Girardello and A. Van Proeyen, Nucl. Phys. B 212, 413 (1983).

[23] L. Randall and R. Sundrum, Nucl. Phys. B 557, 79 (1999);

G. F. Giudice, M. A. Luty, H. Murayama and R. Rattazzi, JHEP 9812, 027 (1998);

J. A. Bagger, T. Moroi and E. Poppitz, JHEP 0004, 009 (2000).

[24] G. Lopes Cardoso and B. A. Ovrut, Nucl. Phys. B 418, 535 (1994). I. L. Buchbinder and S. M. Kuzenko, Phys. Lett. B 202, 233 (1988).

[25] J. A. Bagger, T. Moroi and E. Poppitz, in [23].

[26] J. A. Bagger, T. Moroi and E. Poppitz, Nucl. Phys. B 594, 354 (2001).

[27] M. Ibe, Y. Nakayama and T. T. Yanagida, arXiv:hep-ph/0703110.

[28] M. Luty and R. Sundrum, Phys. Rev. D 67, 045007 (2003).

[29] K. I. Izawa and T. Yanagida, Phys. Lett. B 393, 331 (1997).

[30] T. Asaka, K. Hamaguchi, M. Kawasaki and T. Yanagida, Phys. Rev. D 61, 083512 (2000); V. N. Senoguz and Q. Shafi, Phys. Lett. B 596, 8 (2004).

[31] E. J. Copeland, A. R. Liddle, D. H. Lyth, E. D. Stewart and D. Wands, Phys. Rev. D 49, 6410 (1994); G. R. Dvali, Q. Shafi and R. K. Schaefer, Phys. Rev. Lett. 73, 1886 (1994);

A. D. Linde and A. Riotto, Phys. Rev. D 56, 1841 (1997).

[32] G. Lazarides and C. Panagiotakopoulos, Phys. Rev. D 52, 559 (1995).

[33] M. Kawasaki, M. Yamaguchi and T. Yanagida, Phys. Rev. Lett. 85, 3572 (2000); Phys. Rev. D 63, 103514 (2001).

[34] M. Kawasaki, K. Kohri and T. Moroi, Phys. Lett. B 625, 7 (2005); Phys. Rev. D 71, 083502 (2005).

[35] K. Kohri, T. Moroi and A. Yotsuyanagi, Phys. Rev. D 73, 123511 (2006).

[36] K. Jedamzik, Phys. Rev. D 74, 103509 (2006).

[37] T. Moroi, H. Murayama and M. Yamaguchi, Phys. Lett. B 303, 289 (1993).

[38] M. Bolz, W. Buchmuller and M. Plumacher, Phys. Lett. B 443, 209 (1998). 
[39] M. Bolz, A. Brandenburg and W. Buchmuller, Nucl. Phys. B 606, 518 (2001).

[40] J. R. Ellis, K. A. Olive, Y. Santoso and V. C. Spanos, Phys. Lett. B 588, 7 (2004); L. Roszkowski, R. Ruiz de Austri and K. Y. Choi, JHEP 0508, 080 (2005); D. G. Cerdeno, K. Y. Choi, K. Jedamzik, L. Roszkowski and R. Ruiz de Austri, JCAP 0606, 005 (2006).

[41] F. D. Steffen, JCAP 0609, 001 (2006).

[42] J. Pradler and F. D. Steffen, Phys. Rev. D 75, 023509 (2007); Phys. Lett. B 648, 224 (2007).

[43] I. Affleck, M. Dine and N. Seiberg, Phys. Lett. B 137, 187 (1984); Phys. Lett. B 140, 59 (1984).

[44] I. Affleck, M. Dine and N. Seiberg, Phys. Rev. Lett. 52, 1677 (1984); Nucl. Phys. B 256, 557 (1985).

[45] H. Murayama, Phys. Lett. B 355, 187 (1995).

[46] D. Lindley, Astrophys. J. 294 (1985) 1; M. Y. Khlopov and A. D. Linde, Phys. Lett. B 138, 265 (1984); F.Balestra et al., Sov. J. Nucl. Phys. 39, 626 (1984); M. Yu. Khlopov, Yu. L. Levitan, E. V. Sedelnikov and I. M. Sobol, Phys. Atom. Nucl. 571393 (1994); J. R. Ellis, J. E. Kim and D. V. Nanopoulos, Phys. Lett. B 145, 181 (1984). R. Juszkiewicz, J. Silk and A. Stebbins, Phys. Lett. B 158, 463 (1985); J. R. Ellis, D. V. Nanopoulos and S. Sarkar, Nucl. Phys. B 259 (1985) 175; J. Audouze, D. Lindley and J. Silk, Astrophys. J. 293, L53 (1985); D. Lindley, Phys. Lett. B 171 (1986) 235; M. Kawasaki and K. Sato, Phys. Lett. B 189, 23 (1987); R. J. Scherrer and M. S. Turner, Astrophys. J. 331 (1988) 19; J. R. Ellis et al., Nucl. Phys. B 373, 399 (1992).

[47] M. Kawasaki and T. Moroi, Prog. Theor. Phys. 93, 879 (1995),

[48] R. J. Protheroe, T. Stanev and V. S. Berezinsky, Phys. Rev. D 51, 4134 (1995).

[49] E. Holtmann, M. Kawasaki, K. Kohri and T. Moroi, Phys. Rev. D 60, 023506 (1999).

[50] K. Jedamzik, Phys. Rev. Lett. 84, 3248 (2000).

[51] M. Kawasaki, K. Kohri and T. Moroi, Phys. Rev. D 63, 103502 (2001).

[52] K. Kohri, Phys. Rev. D 64 (2001) 043515.

[53] R. H. Cyburt, J. R. Ellis, B. D. Fields and K. A. Olive, Phys. Rev. D 67, 103521 (2003).

[54] F. Takahashi, arXiv:0705.0579 [hep-ph].

[55] M. Viel, J. Lesgourgues, M. G. Haehnelt, S. Matarrese and A. Riotto, Phys. Rev. D 71, 063534 (2005).

[56] K. Kumekawa, T. Moroi and T. Yanagida, Prog. Theor. Phys. 92, 437 (1994). 
[57] M. Ibe, K. I. Izawa, Y. Shinbara and T. T. Yanagida, Phys. Lett. B 637, 21 (2006).

[58] G. R. Dvali, G. Lazarides and Q. Shafi, Phys. Lett. B 424, 259 (1998).

[59] M. Bastero-Gil, S. F. King and Q. Shafi, arXiv:hep-ph/0604198.

[60] M. ur Rehman, V. N. Senoguz and Q. Shafi, Phys. Rev. D 75, 043522 (2007).

[61] R. A. Battye, B. Garbrecht and A. Moss, JCAP 0609, 007 (2006).

[62] N. Bevis, M. Hindmarsh, M. Kunz and J. Urrestilla, arXiv:astro-ph/0702223.

[63] V. N. Senoguz and Q. Shafi, Phys. Rev. D 71, 043514 (2005).

[64] T. Asaka, K. Hamaguchi, M. Kawasaki and T. Yanagida, Phys. Lett. B 464, 12 (1999); in Ref. [30].

[65] A. D. Linde, Phys. Lett. B 129, 177 (1983).

[66] L. Connors, A. J. Deans and J. S. Hagelin, Phys. Lett. B 220, 368 (1989); L. Connors, A. J. Deans and J. S. Hagelin, Phys. Rev. Lett. 71, 4291 (1993).

[67] S. Kasuya, T. Moroi and F. Takahashi, Phys. Lett. B 593, 33 (2004).

[68] R. Allahverdi, K. Enqvist, J. Garcia-Bellido and A. Mazumdar, Phys. Rev. Lett. 97, 191304 (2006).

[69] D. H. Lyth, JCAP 0704, 006 (2007).

[70] H. Murayama, H. Suzuki, T. Yanagida and J. Yokoyama, Phys. Rev. Lett. 70, 1912 (1993); Phys. Rev. D 50, 2356 (1994).

[71] D. H. Lyth and E. D. Stewart, Phys. Rev. D 53, 1784 (1996).

[72] M. Kawasaki and F. Takahashi, Phys. Lett. B 618, 1 (2005).

[73] E. D. Stewart, M. Kawasaki and T. Yanagida, Phys. Rev. D 54, 6032 (1996).

[74] S. Kasuya, M. Kawasaki and F. Takahashi, Phys. Rev. D 65, 063509 (2002).

[75] D. h. Jeong, K. Kadota, W. I. Park and E. D. Stewart, JHEP 0411, 046 (2004).

[76] M. Kawasaki and K. Nakayama, JCAP 0702, 002 (2007).

[77] M. Endo, F. Takahashi and T. T. Yanagida, in preparation.

[78] L. Kofman, A. D. Linde and A. A. Starobinsky, Phys. Rev. Lett. 73, 3195 (1994); Phys. Rev. D 56, 3258 (1997).

[79] R. Kallosh, L. Kofman, A. D. Linde and A. Van Proeyen, Phys. Rev. D 61, 103503 (2000);

G. F. Giudice, I. Tkachev and A. Riotto, JHEP 9908, 009 (1999);

G. F. Giudice, A. Riotto and I. Tkachev, JHEP 9911, 036 (1999);

R. Kallosh, L. Kofman, A. D. Linde and A. Van Proeyen, Class. Quant. Grav. 17, 4269 (2000) 
[Erratum-ibid. 21, 5017 (2004)].

[80] H. P. Nilles, M. Peloso and L. Sorbo, Phys. Rev. Lett. 87, 051302 (2001); JHEP 0104, 004 (2001).

[81] R. Allahverdi, M. Bastero-Gil and A. Mazumdar, Phys. Rev. D 64, 023516 (2001).

[82] N. Shuhmaher, arXiv:hep-ph/0703319. 